\title{
A NEW MULTIDIMENSIONAL GRAPHICAL APPROACH FOR MATHEMATICS AND PHYSICS
}

\section{Mario Arturo Ruiz Estrada}

Faculty of Economics and Administration (FEA), University of Malaya (UM), Kuala Lumpur, Malaysia *E-mail: marioarturoruiz@gmail.com(Corresponding author)

Homepage: www.econographication.com

\begin{abstract}
This research proposes a serial of new mathematic and physics theoretical framework under a multidimensional focus. The main objective of this paper is to motivate scientists in various areas to use multidimensional coordinate spaces to complement the 2-Dimensional and 3-Dimensional coordinate systems. Additionally, this paper is divided into two large sections that is the mathematic and physics section respectively. Finally, these two sections will be sectioned into eight different elemental theoretical frameworks. The mathematic section is divided into five elemental theoretical frameworks: (i) the idea on zero and infinity in multidimensional coordinate systems; (ii) the megasfinity; (iii) multidimensional partial differentiation; (iv) possibility of plotting matrices on multidimensional coordinate systems; and (v) the global crisis smash graphic effect model (GCSGE-Model). The physics section is divided into three elemental theoretical frameworks: (i) the theory of empty spaces; (ii) the mega time-space continuum; (iii) the Megasverses.
\end{abstract}

(Keywords: Multidimensional graphs, multidimensional graphical modeling, multidimensional coordinate spaces.)

\section{INTRODUCTION}

This research shows a set of new theoretical frameworks from a mathematical and physical viewpoint. Hence, we want to facilitate insight into any complex and dynamic behavior of phenomena from a multidimensional perspective. This paper is divided into two large sections as follows:

\section{Mathematics Section}

Initially, this research will demonstrate graphically and mathematically the case of the multi-dimensional graphical modeling. The idea about zero and infinity is different from those utilizing the traditional 1-dimensional, 2dimensional and 3-dimensional graphical modeling respectively.

Secondly, a new number that is called "megasfinity" ( $)$ will be proposed. The megasfinity is an alternative number which can be used to analyze the different spaces within the multidimensional coordinate systems (Ruiz Estrada, 2011a). This section proposes the infinity coordinate system. The infinity coordinate system visualizes a large number of general-spaces, sub-spaces, micro-spaces, and dimensions (e.g. general-dimensions, subdimensions, and micro-dimensions).

In the third part, this work proposes to introduce the theoretical framework of the multidimensional partial differentiation. This technique makes it possible to build the real time effect for any multidimensional models. The multidimensional partial differentiation makes it possible to simulate infinitesimal possible changes between a large number of multidependent and multi-independent variables that are interacting in different graphical spaces or dimensions. Therefore, the effect of real time in any multidimensional graphical modeling requires the application of multidimensional partial derivatives into different graphical spaces or dimensions.

In the fourth part, this research studies the possibility of plotting matrices in multidimensional coordinate systems? This section of proposes two types of multidimensional coordinate systems to plot matrices. These two types of multidimensional coordinate systems are the surface mapping coordinate system and the four-Dimensional coordinate system. The idea is to represent a large system of equations in the same graphical space and time. 
Finally, the last part of this work presents a theoretical framework for the use of the global crisis smash graphical effect model to evaluate the final effects of a possible global crisis. We present different scenarios and results according to different levels of devastation than any global crisis can generate using any natural scenario. The global crisis smash graphic effect model requires the use of the real time effect on the multidimensional graphical modeling that can facilitate the visualization of different scenarios to evaluate the final impact of any global crisis.

\section{Physics Section}

Initially, the physics section starts with a short introduction on empty space based on a basic mathematical and a graphical modeling respectively. The first part of this section reviews the literature on dimensions and coordinates systems (the Cartesian plane and coordinate space). The second part of this topic will present an introduction to the theory of empty space.

The second part provides a brief review of the general theory of relativity put forward by Albert Einstein from a geometrical perspective. The same research also proposes an alternative coordinate system to study time and space from a multidimensional perspective, entitled "The Mega Time-Space Continuum." This paper also proposes to review and expand the Minkowski four-dimensional space-time ("World") concept. The main objective is to propose an alternative geometrical framework to be applied throughout the study of unknown dimensions in the same graphical space from a multidimensional perspective. Additionally, the same research will demonstrate ing the existence of unknown dimensions through the use of multidimensional coordinate systems.

The final part in the physics section, a new view is proposed about the universe. The Megasverses is a mega-space formed by a number of megasfinity universes (sub-spaces). To have a better understanding about the Megasverses, this research suggests the application of the mega time-space continuum coordinate system framework to explain how distinct universes are located and interrelated within different parts of the Megasverses. In fact, this topic in physics proposes a basic theoretical framework about the gravitation within the Megasverses. Originally, the Megasverses can be considered a mega-space that is formed by megasfinity spaces. Therefore, the idea about the gravitation of the Megasverses is directly connected to three basic conditions: the multidimensional geodesic position, mass and sustainable kinetic energy auto-generative. The endmost condition is a derivative of the last two conditions.

\section{MATHEMATICS SECTION}

\section{The idea of zero about infinity in the multidimensional coordinate systems}

\section{Introduction}

Normally, the idea of the number zero " 0 " is generally seen from a mathematical point of view: zero is considered as the division between positive integers and negative integers from a 1dimensional, 2-dimensional and 3-dimensional graphical perspective (see Figure 1). Meanwhile, the number zero " 0 " does not usually represent any value, and in the end, it often becomes nothing more than a mental refraction in our minds to explain the meaning of nothing. However, from our point of view, the number zero " 0 " is a single real number plotted along the axes of 1-dimensional, 2-dimensional and 3dimensional graphical space, but in this case, it is applied to a multi-dimensional graphical space. We can observe that the number zero " 0 " becomes a large single surface (see Figure 2); this surface shows clearly an infinity of zeros are displayed in all spaces in the mega-space. Moreover, the concept of infinity under multidimensional space changes to a megasfinity. This is equal to the infinite exponential of infinity (or infinity times infinity). See Expression 1:

$$
\infty^{\infty}=\phi
$$

On the other hand, this research demonstrates that these fundamental ideas about the number zero " 0 " change when we are starting to use from 4-dimensional graphical spaces to infinitedimensional (see Ruiz Estrada, 2007). This change in perception is simply that zero is now represented by a single and huge large surface. Conversely, in the case of 1-dimensional, 2dimensional and 3-dimensional graphic modeling, the number zero " 0 " is a fixed single plotted point within its axes. Hence, in the case of multi-dimensional graphic modeling, it is possible to observe the number zero " 0 " in each $\mathrm{Y}_{\mathrm{ij}}$ within its respective mega-space (See Figure 2). 


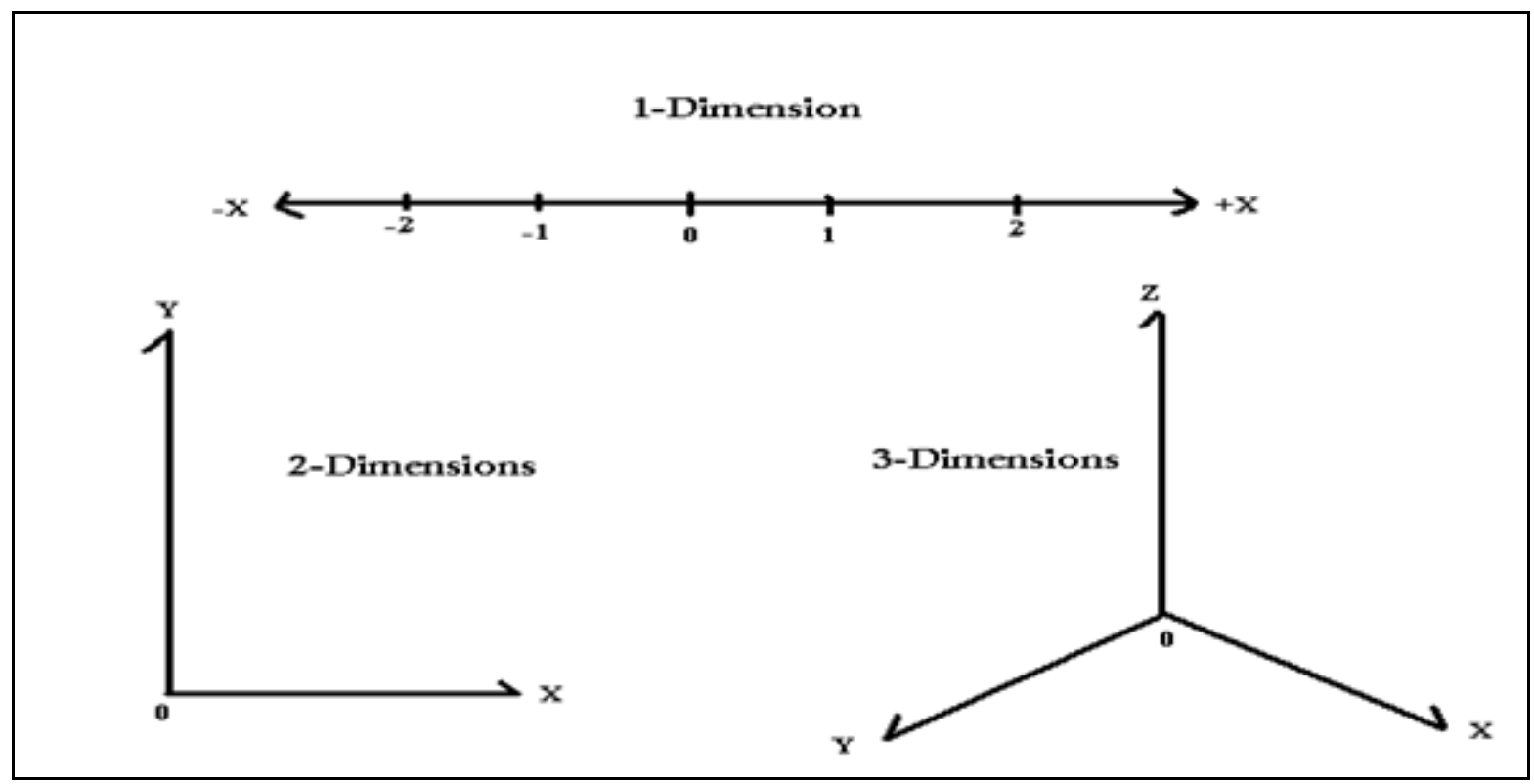

Figure 1: The One-Dimensional, Two-Dimensional and Three-Dimensional Graphical Modeling

\section{The Mega-System Graphical Modeling}

The original function of mega-space graphical modeling is shown by Expression 2.

$$
\mathrm{S}_{\mathrm{i}} \equiv \mathrm{Y}_{\mathrm{i}}=f\left(\mathrm{X}_{\mathrm{i}: \mathrm{j}}\right): \mathrm{i}=\{0,1,2, \ldots, \infty \ldots \text { \&o }\} \& \mathrm{j}=\{0,1,2, \ldots, \infty \ldots \text { \&o }\}
$$

Basically, we build the mega-space systematically, starting from the space for " 0 " until we get to megasfinity space " \&o" (See joins all $X_{i j}$ together with its $Y_{i j}$ under the application of the interlink axes " $\bar{\tau} "$ concept (See Expression 10).

Expression 2). Each space within the mega-space

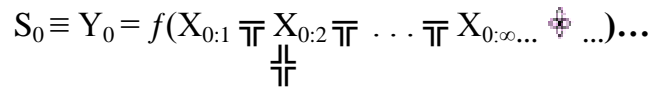

$$
\begin{aligned}
& \mathrm{S}_{1} \equiv \mathrm{Y}_{1}=f\left(\mathrm{X}_{1: 1} \bar{\eta} \mathrm{X}_{1: 2} \bar{\eta} \ldots \bar{\eta} \mathrm{X}_{1: \infty \ldots} \ldots\right) \ldots \\
& \text { 止 }
\end{aligned}
$$

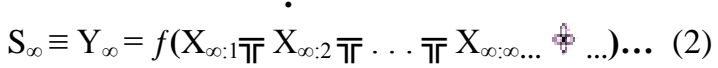

After each function for each space has been built, the next step is to join all spaces simultaneously. This is done by using straight lines to join all $\mathrm{Y}_{\mathrm{ij}}$ until a single, large surface is built. To join each $Y_{i j}$ we require the application of the inter link multilevel space's concept “杰” (see Expression 2 ). In fact, the final mission of the inter link multi-level space's concept is to facilitate the joining of all $\mathrm{Y}_{\mathrm{ji}}$ in the same graphical space. 


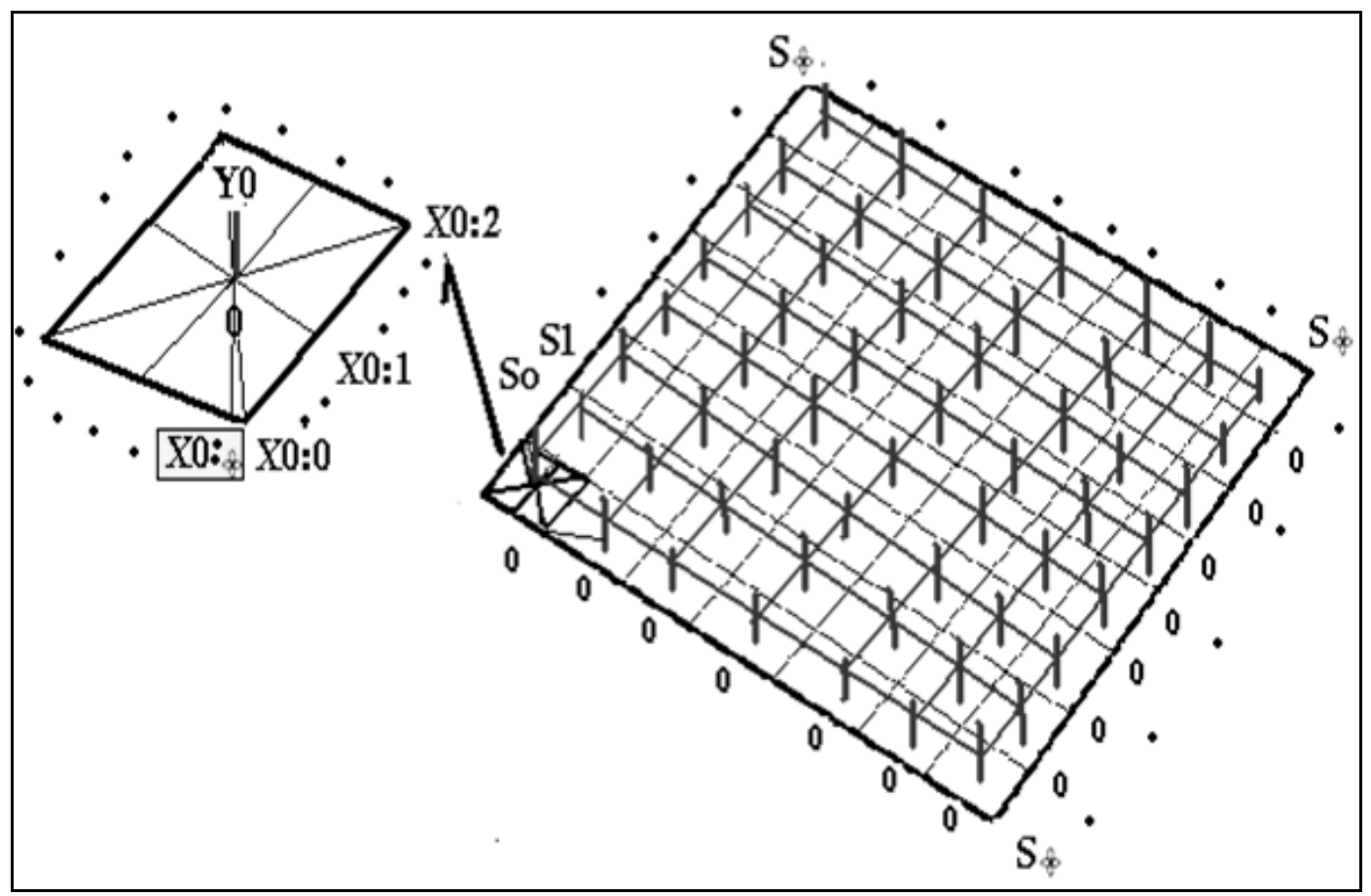

Figure 2: Mega-Space Graphical Modeling

\section{The Megasfinity}

\section{Overview}

For long time, the idea of infinity has mainly been based on a mathematical or philosophical point of view. Usually, infinity is fixed in our mind as that which is unlimited or without end. If we analyze infinity from a geometric perspective, it is possible to observe that the number infinity $(\infty)$ represents a large group of points joined together until we build a geometrical body without end. In fact, the number infinity $(\infty)$ also accounts for the extreme of each axis to represent the idea of continuity without end in any axis when it comes to being 1-Dimensional, 2-Dimensional and 3Dimensional coordinate systems (see Figure 3).

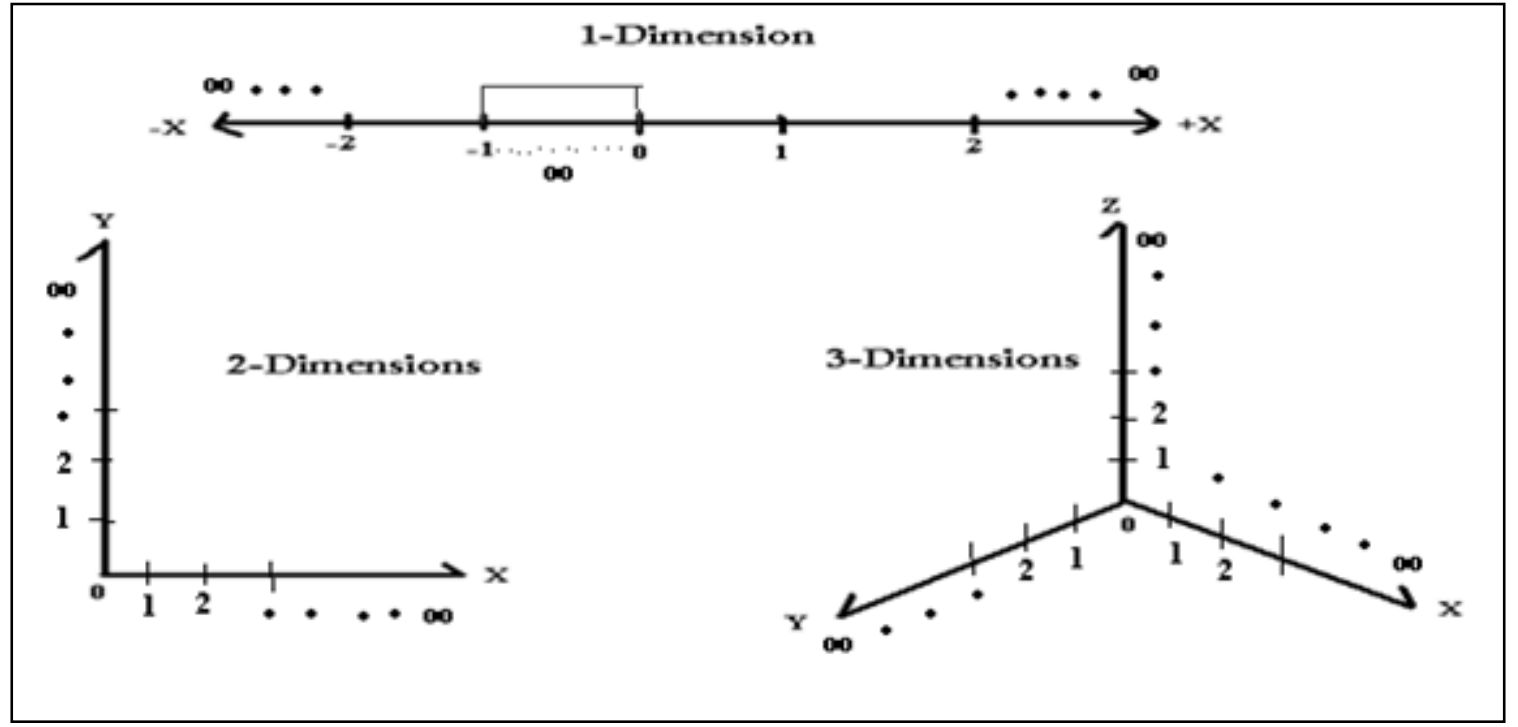

Figure 3: The One-Dimensional, Two-Dimensional and Three-Dimensional Coordinate Systems 
From a multidimensional graphical perspective, it is possible to observe that the fundamental idea of infinity $(\infty)$ changes dramatically because infinity $(\infty)$ cannot explain complex unknown dimensions that exist within multidimensional coordinate systems. Hence, this research suggests a new conceptual number called megasfinity. Megasfinity is equal to infinity to the power of infinity (see Expression 1). Therefore, the idea of infinity will be replaced by megasfinity in the analysis of multidimensional coordinate systems (see Figure 4) to facilitate the analysis of unknown dimensions that cannot be found in 2-dimensional and 3-dimensional coordinate systems.

$$
\infty^{\infty}=
$$

The Megasfinity ( $\phi^{\circ}$ ) will play a very important role to explain unknown geometrical spaces that were never explored before in geometry under the use of an alternative group of Multidimensional coordinate systems. This is made possible by the observation of a large number of axes displayed in different multidimensional coordinate systems. The multidimensional coordinate system that will be used in this research is the infinity coordinate system. In this specific coordinate system, it is possible to observe a megasfinity number of general-spaces (i), sub-spaces (j) and microspaces (k). At the same time, we also assume that different dimensions exist in each general-space, sub-space and micro-space, such as generaldimensions, sub-dimensions and microdimensions respectively. The infinity coordinate system shows its own coordinate system, as in Expression 2.

$$
\left(\alpha_{i: j: k}, \beta_{i: j: k}\right)
$$

The infinity coordinate system starts from the general-space 0 (see Expression 3) until it gets to the general space megasfinity (see Expression 4).

$$
\begin{aligned}
& \mathrm{GS}_{0} \equiv\left(\alpha_{0: 0: 0,}, \beta_{0: 0: 0 \ldots \ldots \ldots} \alpha_{0:} \phi^{\phi}, \beta_{0:} \phi^{\phi}: \phi^{\infty}\right) \\
& \text { GS } \phi \equiv\left(\alpha \phi: 0: 0, \beta \phi_{0: 0 \ldots \ldots . . .} \alpha \phi \phi_{0}: \phi, \beta \phi: \phi: \phi\right)
\end{aligned}
$$

Additionally, we must assume that in the infinity coordinate system, the variable time $(\mathrm{t})$ is running at different speeds in each generaldimension, sub-dimension, micro-dimension and nano-dimension. By dividing time into three categories: constant time (KT) or past time, partial time (PT) or present time, and chaos time (CT) or future time. Hence, each general-space or general-dimension (i), sub-space or subdimension (j) and micro-space or microdimension $(\mathrm{k})$ can experience these three types of time according to Expression 5.

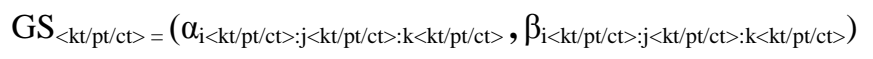

Finally, we can say that the fundamental idea of infinity can change radically when we apply it to the infinity coordinate system. Here, we can observe that there exists a megasfinity of general-spaces (i), sub-spaces (j) and micro- spaces $(\mathrm{k})$ in different spaces within the infinity coordinate system. Additionally, this specific coordinate system graphically shows the idea of space and time from a multidimensional perspective. 


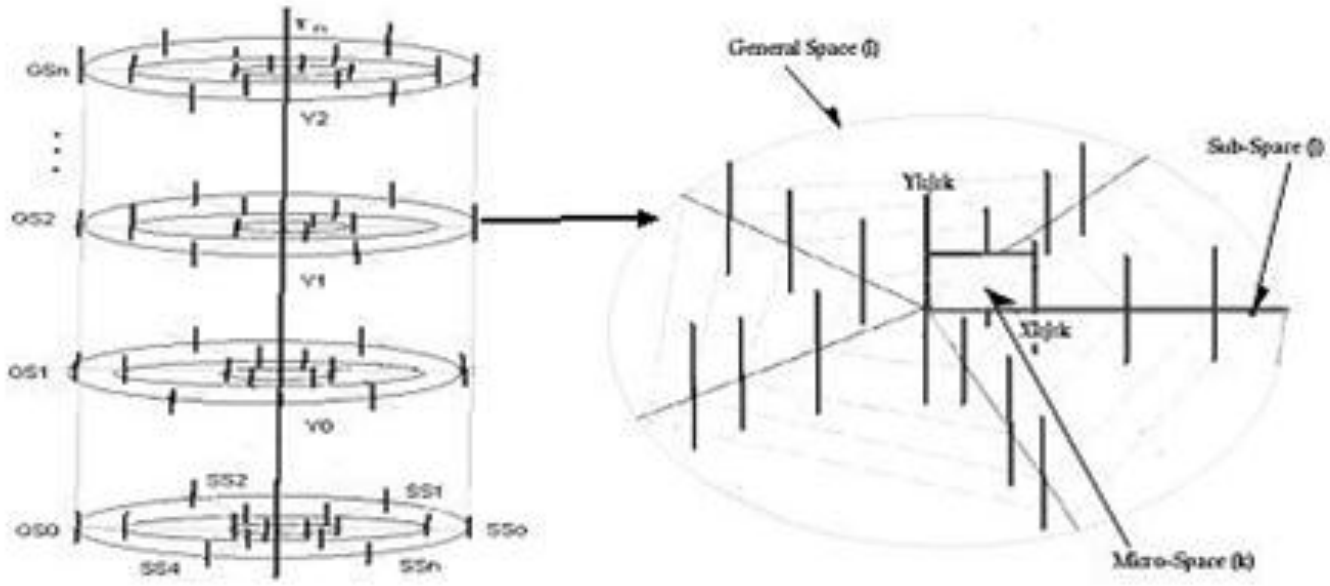

Figure 4: Infinity Coordinate System

\section{Multidimensional partial differentiation}

\section{Introduction to the Multidimensional Partial Differentiation}

This section will introduce the theoretical framework of multidimensional partial differentiation, which is based on different rules and conditions for the uses of multidimensional derivatives in sciences. According to the theoretical framework of multidimensional partial differentiation, the original functions start with Expression 1:

$$
\begin{aligned}
& \mathrm{S}_{\mathrm{i}: \mathrm{j}: \mathrm{k}} \equiv \mathrm{Y}_{\mathrm{i}: \mathrm{j}: \mathrm{k}}=f\left(\mathrm{X}_{\mathrm{i}: \mathrm{j}: \mathrm{k}}\right) \\
& \Delta \mathrm{Y}_{\mathrm{i}: \mathrm{j}: \mathrm{k}} / \Delta \mathrm{X}_{\mathrm{i} \mathrm{j}: \mathrm{k}}=f\left(\Delta \mathrm{X}_{\mathrm{i} \mathrm{j}: \mathrm{k}}\right)-f\left(\mathrm{X}_{\mathrm{i}: \mathrm{j}: \mathrm{k}}\right) / \Delta \mathrm{X}_{\mathrm{i} \mathrm{j}: \mathrm{k}} \\
& f_{\mathrm{i}: \mathrm{j}: \mathrm{k}}=\partial \mathrm{Y}_{\mathrm{i}: \mathrm{j}: \mathrm{k}} / \partial \mathrm{X}_{\mathrm{i}: \mathrm{j}: \mathrm{k}} \equiv \lim \Delta \mathrm{Y}_{\mathrm{i}: \mathrm{j}: \mathrm{k}} / \Delta \mathrm{X}_{\mathrm{i} \mathrm{j}: \mathrm{k}} \\
& \Delta \mathrm{Xi}: \mathrm{j}: \mathrm{k} \rightarrow 0
\end{aligned}
$$

In Expression 1, the variables $\mathrm{X}_{\mathrm{i} \mathrm{j}: \mathrm{k}}$ (generalspaces $(\mathrm{i})=0,1,2, \ldots, \infty \ldots ; \quad$ sub-spaces $(\mathrm{j})=$ $0,1,2, \ldots, \infty \ldots$ and micro-spaces $(\mathrm{k})=$ $0,1,2, \ldots, \infty \ldots)$ are all multi-independent variables and $\mathrm{Y}_{\mathrm{i}: \mathrm{j}: \mathrm{k}}($ general-spaces $(\mathrm{i})=0,1,2, \ldots, \infty \ldots$; subspaces $(\mathrm{j})=0,1,2, \ldots, \infty \ldots$ and micro-spaces $(\mathrm{k})=$ $0,1,2, \ldots, \infty \ldots)$ are all multi-dependent variables. The derivatives from $X_{i, j: k}(i=0,1,2, \ldots, \infty \ldots)$ all remain non-fixed, so there will be corresponding changes in all $\Delta \mathrm{Y}_{\mathrm{i}: \mathrm{j}: \mathrm{k}}$ (see Expression 7). We assume that different partial derivatives of $Y_{\mathrm{i}: \mathrm{j}: \mathrm{k}}$ are held to be changing all the time, simultaneously, with respect to its $X_{\mathrm{i}: \mathrm{j}: \mathrm{k}}$ in different general-spaces (i), sub-spaces (j) and micro-spaces (k). This is based on the application of the Omnis Mobilis assumption by
Ruiz Estrada (2011a). Therefore, $\Delta \mathrm{X}_{\mathrm{i} \mathrm{j}: \mathrm{k}} \rightarrow>0$, so that the limit will constitute a series of multidimensional partial derivatives that interact together in different spaces in the infinity coordinate system. Basically, multidimensional partial differentiation starts with function $f^{\beta}$ 0:0:0, which is located in the general space 0 , subspace 0 and micro-space 0 . This function can be successively derived infinite times $(\beta)$ until the partial derivative " $\infty$ " it is reached (See Expression 4). Each space runs into its specific partial derivative with different relationships

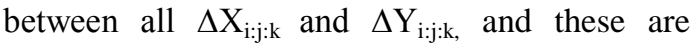
finally joined together by the application of the interlinked sub-axes concept " "Г" (see Expression 16). The application of the interlinked sub-axes 
concept " $\pi "$ is to connect all the partial the same sub-space (j) and general-space (i).

derivatives running in each micro-space $(\mathrm{k})$ into

$\mathrm{S}_{0}{ }^{\prime}=\delta f^{\prime}\left(\mathrm{Y}_{0: 0: 0}\right) / \delta \mathrm{X}_{0: 0: 0} \mathrm{~d}^{\prime} \mathrm{X}_{0: 0: 0} \bar{\tau} \delta f^{\prime}\left(\mathrm{Y}_{0: 0: 1}\right) / \delta \mathrm{X}_{0: 0: 1} \mathrm{~d}^{\prime} \mathrm{X}_{0: 0: 1} \overline{\eta \Gamma} \ldots \delta f^{\prime}\left(\mathrm{Y}_{0: 0: \infty}\right) / \delta \mathrm{X}_{0: 0: \infty} \mathrm{d} \mathrm{X}_{0: 0: \infty}$ $\mathrm{S}_{0} "=\delta f^{\prime \prime}\left(\mathrm{Y}_{0: 0: 0}\right) / \delta \mathrm{X}_{0: 0: 0} \mathrm{~d}^{\prime \prime} \mathrm{X}_{0: 0: 0} \bar{\eta} \delta f^{\prime \prime}\left(\mathrm{Y}_{0: 0: 1}\right) / \delta \mathrm{X}_{0: 0: 1} \mathrm{~d}^{\prime \prime} \mathrm{X}_{0: 0: 1} \bar{\eta} \ldots \delta f^{\prime \prime}\left(\mathrm{Y}_{0: 0: \infty}\right) / \delta \mathrm{X}_{0: 0: \infty} \mathrm{d}^{\prime \prime} \mathrm{X}_{0: 0: \infty}$

$\mathrm{S}_{0}^{\infty}=\delta f^{\infty}\left(\mathrm{Y}_{0: 0: 0}\right) / \delta \mathrm{X}_{0: 0: 0} \mathrm{~d}^{\infty} \mathrm{X}_{0: 0: 0} \overline{7 \Gamma} \delta f^{\infty}\left(\mathrm{Y}_{0: 0: 1}\right) / \delta \mathrm{X}_{0: 0: 1} \mathrm{~d}^{\infty} \mathrm{X}_{0: 0: 1} \overline{7 \Gamma} \ldots \delta f^{\infty}\left(\mathrm{Y}_{0: 0: \infty}\right) / \delta \mathrm{X}_{0: 0: \infty} \mathrm{d}^{\infty} \mathrm{X}_{0: 0: \infty}=0$

$\mathrm{S}_{1}{ }^{\prime}=\delta f^{\prime}\left(\mathrm{Y}_{1: 0: 0}\right) / \delta \mathrm{X}_{1: 0: 0} \mathrm{~d}^{\prime} \mathrm{X}_{1: 0: 0} \overline{\rceil \Gamma} \delta f^{\prime}\left(\mathrm{Y}_{1: 0: 1}\right) / \delta \mathrm{X}_{1: 0: 1} \mathrm{~d}^{\prime} \mathrm{X}_{1: 0: 1} \overline{\rceil \Gamma} \ldots \delta f^{\prime}\left(\mathrm{Y}_{1: 0: \infty}\right) / \delta \mathrm{X}_{1: 0: \infty} \mathrm{d}^{\prime} \mathrm{X}_{1: 0: \infty}$ $\mathrm{S}_{1}=\delta f^{\prime \prime}\left(\mathrm{Y}_{1: 0: 0}\right) / \delta \mathrm{X}_{1: 0: 0} \mathrm{~d}^{\prime \prime} \mathrm{X}_{1: 0: 0} \bar{\eta} \delta f^{\prime \prime}\left(\mathrm{Y}_{1: 0: 1}\right) / \delta \mathrm{X}_{1: 0: 1} \mathrm{~d} " \mathrm{X}_{1: 0: 1} \bar{\eta} \ldots \delta f^{\prime \prime}\left(\mathrm{Y}_{1: 0: \infty}\right) / \delta \mathrm{X}_{1: 0: \infty} \mathrm{d} " \mathrm{X}_{1: 0: \infty}$

$\mathrm{S}_{1}^{\infty}=\delta f^{\infty}\left(\mathrm{Y}_{1: 0: 0}\right) / \delta \mathrm{X}_{1: 0: 0} \mathrm{~d}^{\infty} \mathrm{X}_{1: 0: 0} \bar{\tau} \delta f^{\infty}\left(\mathrm{Y}_{1: 0: 1}\right) / \delta \mathrm{X}_{1: 0: 1} \mathrm{~d}^{\infty} \mathrm{X}_{1: 0: 1} \bar{\tau} \ldots \delta f^{\infty}\left(\mathrm{Y}_{1: 0: \infty}\right) / \delta \mathrm{X}_{1: 0: \infty} \mathrm{d}^{\infty} \mathrm{X}_{1: 0: \infty}=0$

$\mathrm{S}_{2, .,}{ }^{\prime}=\delta f^{\prime}\left(\mathrm{Y}_{2: 0: 0}\right) / \delta \mathrm{X}_{2: 0: 0} \mathrm{~d}^{\prime} \mathrm{X}_{2: 0: 0} \bar{\eta} \delta f^{\prime}\left(\mathrm{Y}_{2: 0: 1}\right) / \delta \mathrm{X}_{2: 0: 1} \mathrm{~d}^{\prime} \mathrm{X}_{2: 0: 1} \overline{7 \Gamma} \ldots \delta f^{\prime}\left(\mathrm{Y}_{2: 0: \infty}\right) / \delta \mathrm{X}_{2: 0: \infty} \mathrm{d} \mathrm{X}_{2: 0: \infty}$

$\mathrm{S}_{2}=\delta f^{\prime \prime}\left(\mathrm{Y}_{2: 0: 0}\right) / \delta \mathrm{X}_{2: 0: 0} \mathrm{~d}^{\prime \prime} \mathrm{X}_{2: 0: 0} \overline{\eta \Gamma} \delta f^{\prime \prime}\left(\mathrm{Y}_{2: 0: 1}\right) / \delta \mathrm{X}_{2: 0: 1} \mathrm{~d} " \mathrm{X}_{2: 0: 1} \overline{\eta \Gamma} \ldots \delta f^{\prime \prime}\left(\mathrm{Y}_{2: 0: \infty}\right) / \delta \mathrm{X}_{2: 0: \infty} \mathrm{d} " \mathrm{X}_{2: 0: \infty}$

$\mathrm{S}_{2}^{\infty}=\delta f^{\infty}\left(\mathrm{Y}_{2: 0: 0}\right) / \delta \mathrm{X}_{2: 0: 0} \mathrm{~d}^{\infty} \mathrm{X}_{2: 0: 0} \bar{\pi} \delta f^{\infty}\left(\mathrm{Y}_{2: 0: 1}\right) / \delta \mathrm{X}_{2: 0: 1} \mathrm{~d}^{\infty} \mathrm{X}_{2: 0: 1} \bar{\pi} \ldots \delta f^{\infty}\left(\mathrm{Y}_{2: 0: \infty}\right) / \delta \mathrm{X}_{2: 0: \infty} \mathrm{d}^{\infty} \mathrm{X}_{2: 0: \infty}=0$

$\mathrm{S}_{\infty}{ }^{\prime}=\delta f^{\prime}\left(\mathrm{Y}_{\infty: 0: 0}\right) / \delta \mathrm{X}_{\infty: 0: 0} \mathrm{~d} \mathrm{X}_{\infty: 0: 0} \overline{\nabla \Gamma} \delta f^{\prime}\left(\mathrm{Y}_{\infty: 0: 1}\right) / \delta \mathrm{X}_{\infty: 0: 1} \mathrm{~d}^{\prime} \mathrm{X}_{\infty: 0: 1} \overline{\nabla \Gamma} \ldots \delta f^{\prime}\left(\mathrm{Y}_{\infty: 0: 0: \infty}\right) / \delta \mathrm{X}_{\infty: 0: \infty} \mathrm{d} \mathrm{X}_{\infty: 0: \infty}$

$\mathrm{S}_{\infty}=\delta f^{\prime \prime}\left(\mathrm{Y}_{\infty: 0: 0}\right) / \delta \mathrm{X}_{\infty: 0: 0} \mathrm{~d} " \mathrm{X}_{\infty: 0: 0} \overline{\nabla \Gamma} \delta f^{\prime \prime}\left(\mathrm{Y}_{\infty: 0: 1}\right) / \delta \mathrm{X}_{\infty: 0: 1} \mathrm{~d} " \mathrm{X}_{\infty: 0: 1} \bar{\eta} \ldots \delta f^{\prime \prime}\left(\mathrm{Y}_{\infty: 0: \infty}\right) / \delta \mathrm{X}_{\infty: 0: \infty} \mathrm{d} " \mathrm{X}_{\infty: 0: \infty}$

$$
\mathrm{S}_{\infty}^{\infty}=\delta f^{\infty}\left(\mathrm{Y}_{\infty: 0: 0}\right) / \delta \mathrm{X}_{\infty: 0: 0} \mathrm{~d}^{\infty} \mathrm{X}_{\infty: 0: 0} \overline{\eta \Gamma} \delta f^{\infty}\left(\mathrm{Y}_{\infty: 0: 1}\right) / \delta \mathrm{X}_{\infty: 0: 1} \cdot \mathrm{d}^{\infty} \mathrm{X}_{\infty: 0: 1} \bar{\tau} \ldots \delta f^{\infty}\left(\mathrm{Y}_{\infty: 0: \infty}\right) / \delta \mathrm{X}_{\infty: 0: \infty} \mathrm{d}^{\infty} \mathrm{X}_{\infty: 0: \infty}=0(4)
$$

After we apply multidimensional partial differentiation upon each micro-space $(\mathrm{k})$ in the identical sub-space (j) and general-space (i), the next step is to join all spaces simultaneously. We proceed to join all the general-spaces (i) into the same infinity coordinate system by the application of interlink multilevel systems “" "th" (see Expression 17). However, we can calculate a large number of partial derivatives in each micro-space $(\mathrm{k})$ in the same sub-space $(\mathrm{j})$ and general space (i) in the infinity coordinate system simultaneously. The idea is to observe the marginal changes of each space within the infinity coordinate system respectively. We assume that each space is going to run an infinite number of multidimensional partial derivatives simultaneously until it becomes equal to 0 . If we start to apply the first multidimensional derivative from general-space 0 up to the general-space megasfinity simultaneously, then each multidimensional derivative is going to exhibit different behavior in time and space

$$
\begin{aligned}
& \mathrm{dy}_{\mathrm{i}: \mathrm{j}: \mathrm{k}} / \mathrm{dx}_{\mathrm{i}: \mathrm{j}: \mathrm{k}}=0 \text { or } f^{\prime}\left(\mathrm{x}_{\mathrm{i}: \mathrm{j}: \mathrm{k}}\right)=0 \\
& \mathrm{~d} / \mathrm{dx}_{\mathrm{i}: \mathrm{j} \cdot \mathrm{k}}=\mathrm{nx}{ }^{\mathrm{n}-1}{ }_{\mathrm{i}: \mathrm{j} \cdot \mathrm{k}} \text { or } f^{\prime}\left(\mathrm{x}_{\mathrm{i}: \mathrm{j} \mathrm{k}}\right)=\mathrm{nx} \mathrm{x}_{\mathrm{i} \mathrm{i} \cdot \mathrm{j} \cdot \mathrm{k}} \\
& \mathrm{d} / \mathrm{dcx}_{\mathrm{i}: \mathrm{j}: \mathrm{k}}=\operatorname{cnn} \mathrm{x}^{\mathrm{n}-1} \text { i:j:k } \text { or } f^{\prime}\left(\mathrm{x}_{\mathrm{i}: \mathrm{j}: \mathrm{k}}\right)=\mathrm{cnx} \mathrm{x}^{\mathrm{n}-1} \mathrm{i} \mathrm{j}: \mathrm{k}
\end{aligned}
$$

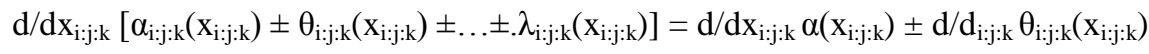

$$
\begin{aligned}
& \pm \ldots \pm . \lambda_{\mathrm{i}: \mathrm{j}: \mathrm{k}}\left(\mathrm{x}_{\mathrm{i}: \mathrm{j}: \mathrm{k}}\right) \text { or } \alpha^{\prime}\left(\mathrm{x}_{\mathrm{i} \mathrm{j}: \mathrm{k}}\right) \pm \theta^{\prime}\left(\mathrm{x}_{\mathrm{i} \mathrm{j}: \mathrm{k}}\right) \pm \ldots \pm . \lambda^{\prime}\left(\mathrm{x}_{\mathrm{i} \mathrm{j}: \mathrm{k}}\right)
\end{aligned}
$$

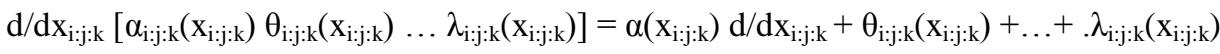

$$
\begin{aligned}
& \alpha\left(\mathrm{x}_{\mathrm{i}: \mathrm{j}: \mathrm{k}}\right)+\theta_{\mathrm{i} \mathrm{j}: \mathrm{k}}\left(\mathrm{x}_{\mathrm{i}: \mathrm{j}: \mathrm{k}}\right) \mathrm{d} / \mathrm{dx}_{\mathrm{i}: \mathrm{j}: \mathrm{k}}+\ldots+. \lambda_{\mathrm{i}: \mathrm{j}: \mathrm{k}}\left(\mathrm{x}_{\mathrm{i}: \mathrm{j}: \mathrm{k}}\right)
\end{aligned}
$$




$$
\begin{aligned}
& \alpha\left(\mathrm{x}_{\mathrm{i}: \mathrm{j} \mathrm{k}}\right)+\theta_{\mathrm{i}: \mathrm{j}: \mathrm{k}}\left(\mathrm{x}_{\mathrm{i}: \mathrm{j}: \mathrm{k}}\right)+\ldots+. \lambda_{\mathrm{i} \mathrm{j} \mathrm{j} \mathrm{k}}\left(\mathrm{x}_{\mathrm{i} \mathrm{j}: \mathrm{k}}\right) \mathrm{d} / \mathrm{dx}_{\mathrm{i}: \mathrm{j}: \mathrm{k}}
\end{aligned}
$$

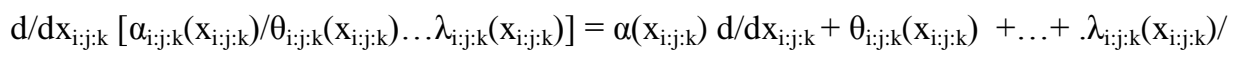

$$
\begin{aligned}
& {\left[\theta_{\mathrm{i}: \mathrm{j}: \mathrm{k}}\left(\mathrm{x}_{\mathrm{i}: \mathrm{j}: \mathrm{k}}\right)+\ldots+\lambda_{\mathrm{i}: \mathrm{j}: \mathrm{k}}\left(\mathrm{x}_{\mathrm{i} \mathrm{j} \mathrm{j} \mathrm{k}}\right)\right]^{2}}
\end{aligned}
$$

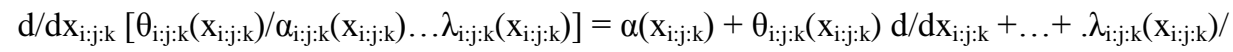

$$
\begin{aligned}
& {\left[\alpha_{\mathrm{i}: \mathrm{j}: \mathrm{k}}\left(\mathrm{x}_{\mathrm{i}: \mathrm{j}: \mathrm{k}}\right)+\ldots+\lambda_{\mathrm{ij: \textrm {k }}}\left(\mathrm{x}_{\mathrm{i} \mathrm{j}: \mathrm{k}}\right)\right]^{2}}
\end{aligned}
$$

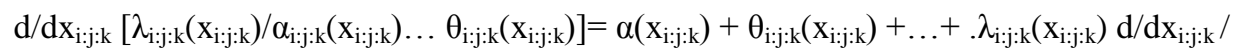

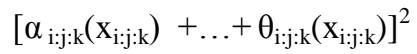

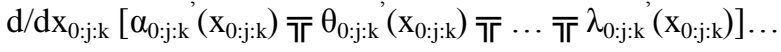

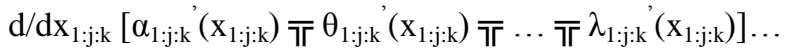

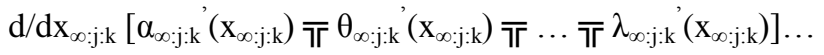

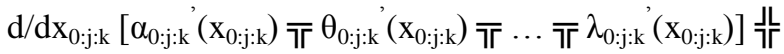

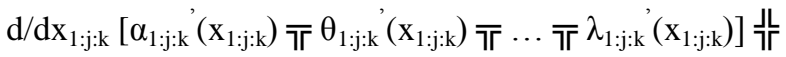

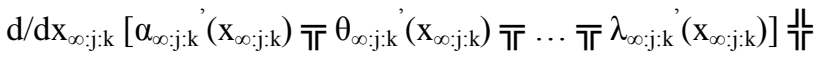

\section{Is It Possible to Plot Matrices in the Multidimensional Coordinate Systems?}

\section{The Matrix Theory from a Multidimensional Graphical View}

In the process to plot matrices, we suggest the application of two types of coordinate systems: the mega-surface mapping coordinate system and the four-Dimensional coordinate system. Both coordinate systems make it possible to plot matrix "m" by " $n$ " at the same graphical space and time. The first coordinate system for plotting matrices is called the mega-surface mapping coordinate system. This coordinate system is represented by a large surface that starts from A11 to A1n on the first horizontal level of the mega-surface mapping coordinate system. The second horizontal level of the mega-surface mapping coordinate system starts from A21 to A2n. This follows sequentially onwards until we reach the infinite horizontal level of the megasurface mapping coordinate system, which starts from Am1 to Amn. We would like to mention that each Aij is a vertical line or axis; at the same time, each Aij is divided by positive (+) and negative (-) values. The division between the positive and negative values of each Aij is by the number zero. Therefore, if we see all Aij together, then we can observe that zero is a large surface in the middle part of the mega-surface mapping coordinate system. If we plot all Aij into the mega-surface mapping coordinate system from A11 to Amn, then we can proceed to join all Aij by straight lines until we can build a single, large surface within the mega-surface mapping coordinate system (see Figure 5).

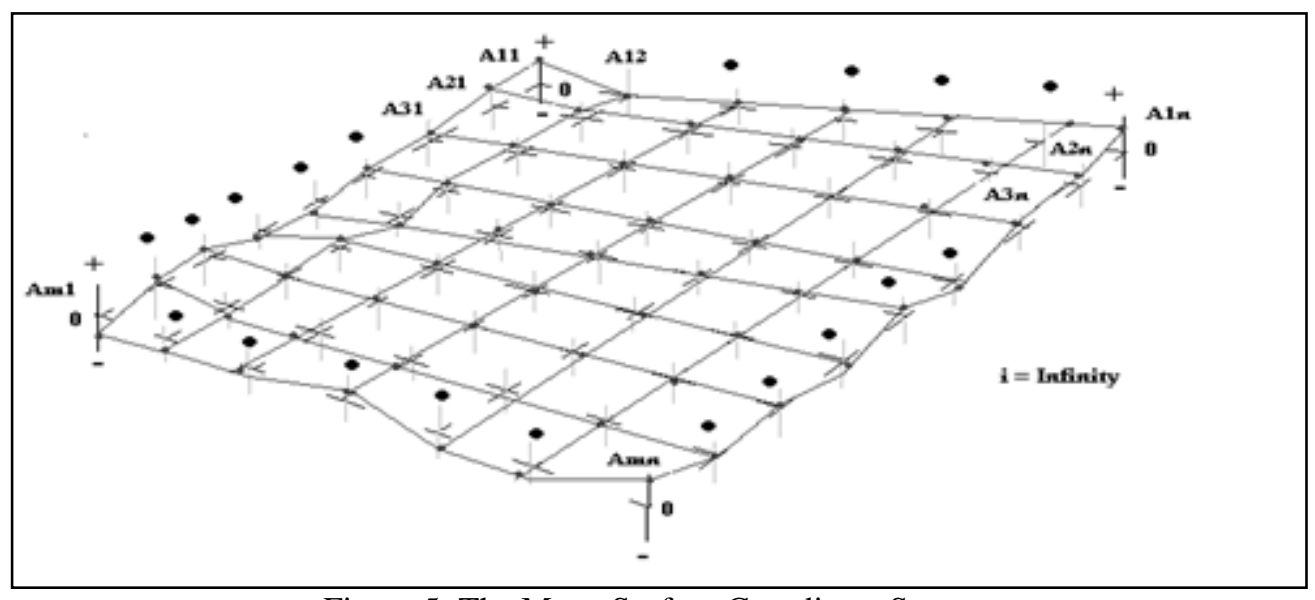

Figure 5: The Mega-Surface Coordinate System 
To plot matrix operations such as addition, subtraction and multiplication, we suggest the use of three mega-surface coordinate systems from a general approach, separately. We start by plotting the two first large matrices from the matrix $-A_{i j}$ and the matrix $-B_{i j}$. These two matrices are plotted into its mega-surface coordinate system from a general approach respectively, and finally; we build our third surface (matrix $-\mathrm{C}_{\mathrm{ij}}$ ) based on the final result from the addition, subtraction or multiplication of the matrix $-\mathrm{A}_{\mathrm{ij}}$ and the matrix- $\mathrm{B}_{\mathrm{ij}}$ (see Figure 6).

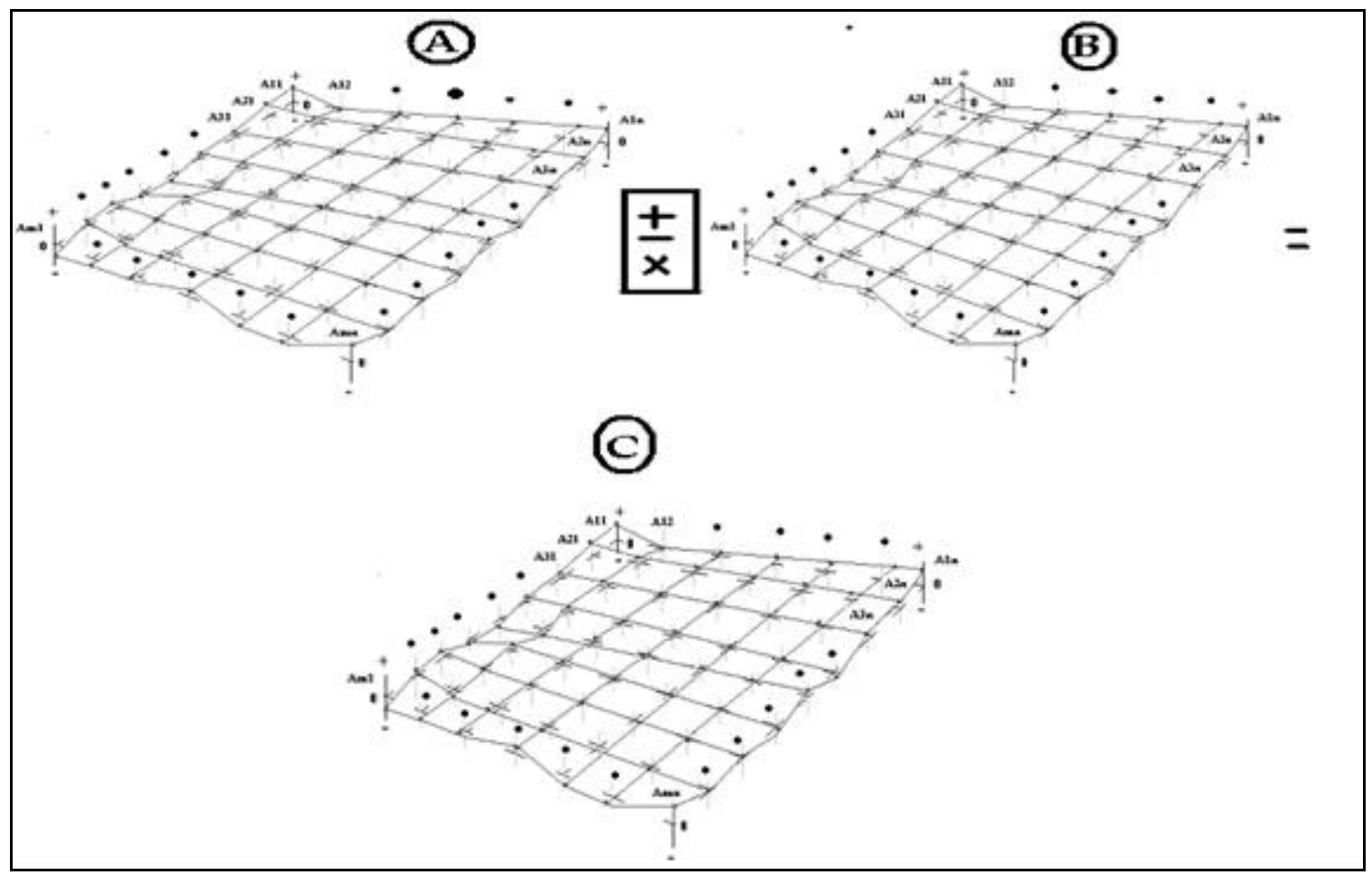

Figure 6: The Mega-Surface Coordinate System Applied on Addition, Subtraction and Multiplication of Matrix

For example, Figure 7 demonstrates how we can plot the sum of two matrices (matrix- $\mathrm{A}_{\mathrm{ij}}$ and matrix- $\left.\mathrm{B}_{\mathrm{ij}}\right)$, and the final result which is plotted on matrix- $\mathrm{C}_{\mathrm{ij}}$ (see Figure 7)

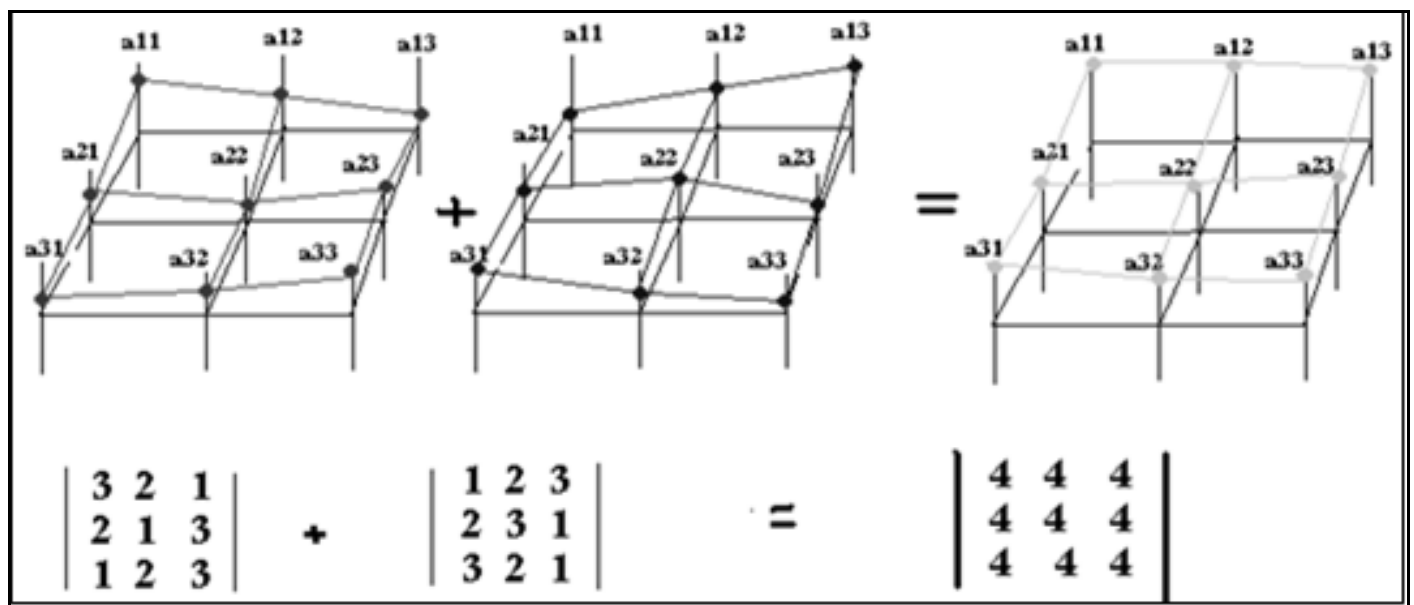

Figure 7: The plotting of the Sum of two Matrices on the Application of the Mega-Surface Coordinate System in General Approach 
The second coordinate system which we will use for plotting a matrix is based on the application of the 4-dimensional coordinate system. The idea is to plot the determinant and the final product terms. We are considering a three-order three by

\section{Left product terms}

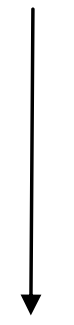

three determinant matrix. We assume that the determinant is a uniquely defined scalar number related to any matrix. Initially, we have a thirdorder determinant matrix (see Expression 1).

Right product terms

\begin{tabular}{|ccc|}
$\mathrm{a}_{11}$ & $\mathrm{a}_{12}$ & $\mathrm{a}_{13}$ \\
$\mathrm{a}_{21}$ & $\mathrm{a}_{22}$ & $\mathrm{a}_{23}$ \\
$\mathrm{a}_{31}$ & $\mathrm{a}_{32}$ & $\mathrm{a}_{33}$ \\
\hline $\mathrm{a}_{11}$ & $\mathrm{a}_{12}$ & $\mathrm{a}_{13}$ \\
$\mathrm{a}_{21}$ & $\mathrm{a}_{22}$ & $\mathrm{a}_{23}$
\end{tabular}

The six product terms are distributed by right and left product terms.

First, the right product terms are formed by $\mathrm{V}_{1}$, $\mathrm{V}_{2}$ and $\mathrm{V}_{3}$ :

$$
\begin{aligned}
& \mathrm{V}_{1}=\mathrm{a}_{11} \quad \mathrm{a}_{22} \quad \mathrm{a}_{33} \\
& \mathrm{~V}_{2}=\mathrm{a}_{21} \quad \mathrm{a}_{32} \mathrm{a}_{13} \\
& \mathrm{~V}_{3}=\mathrm{a}_{31} \mathrm{a}_{12} \mathrm{a}_{23}
\end{aligned}
$$

Second, the left product terms are formed by $\mathrm{V}_{4}$, $\mathrm{V}_{5}$ and $\mathrm{V}_{6}$ :

$$
\begin{aligned}
& \mathrm{V}_{4}=\mathrm{a}_{31} \mathrm{a}_{22} \mathrm{a}_{13} \\
& \mathrm{~V}_{5}=\mathrm{a}_{11} \mathrm{a}_{32} \mathrm{a}_{23} \\
& \mathrm{~V}_{6}=\mathrm{a}_{21} \quad \mathrm{a}_{12} \mathrm{a}_{33}
\end{aligned}
$$

In the same matrix $3 \times 3$ model, we have two final products formed by $R_{1}$ and $R_{2}$ :

$$
\begin{aligned}
& \mathrm{R}_{1}=\mathrm{V}_{1}+\mathrm{V}_{2}+\mathrm{V}_{3} \\
& \mathrm{R}_{2}=\mathrm{V}_{4}+\mathrm{V}_{5}+\mathrm{V}_{6}
\end{aligned}
$$

Finally, we measure the determinant " $\Delta$ " based on the difference between $\mathrm{R}_{1}$ and $\mathrm{R}_{2}$ :

$$
\Delta=\left(\mathrm{R}_{1}\right)-\left(\mathrm{R}_{2}\right)
$$

In the process to plot the determinant and three product terms respectively into the 4dimensional coordinate system, we suggest finding $\mathrm{F}_{1}{ }_{1}, \mathrm{~F}_{2}{ }_{2}$ and $\mathrm{F}^{*}{ }_{3}$ (see Expression 11,12 and 13):

$$
\begin{aligned}
& \mathrm{F}_{1}^{*}=\mathrm{V}_{1}-\mathrm{V}_{4} \\
& \mathrm{~F}_{2}^{*}=\mathrm{V}_{2}-\mathrm{V}_{5} \\
& \mathrm{~F}_{3}^{*}=\mathrm{V}_{3}-\mathrm{V}_{6}
\end{aligned}
$$

In the final process of plotting a matrix, we suggest plotting $\mathrm{F}_{1}{ }_{1}, \mathrm{~F}_{2}{ }_{2}$ and $\mathrm{F}_{3}{ }_{3}$ into the axes $\left(\mathrm{X}_{1}, \mathrm{X}_{2}\right.$ and $\left.\mathrm{X} 3\right)$, where the determinant " $\Delta$ " needs to be plotted on the " $Y$ ", axis in the 4dimensional coordinate system (see Figure 8). Once we plot each axis $\mathrm{F}_{1}{ }_{1}, \mathrm{~F}^{*}{ }_{2}$ and $\mathrm{F}_{3}{ }_{3}$ and the determinant " $\Delta$ " into the 4-dimensional coordinate system, then we can proceed to join $\mathrm{F}_{1}{ }_{1}, \mathrm{~F}_{2}{ }_{2}$ and $\mathrm{F}_{3}{ }_{3}$ and the determinant " $\Delta$ " by straight lines together until we build a single surface. The final determinant that is represented by the symbol " $\Delta$ ", it is calculated on the uses of the Expression 14:

$$
\Delta=\mathrm{F}_{1}^{*}+\mathrm{F}_{2}^{*}+\mathrm{F}_{3}^{*}
$$

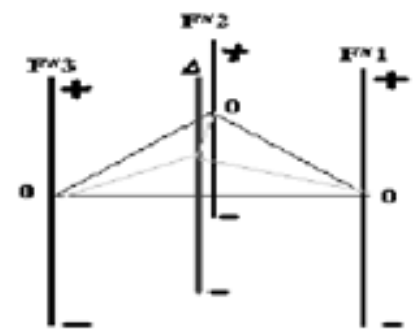

Figure 8: The Four Dimensional Coordinate System 
Our second short example is a simple three by three matrix that we will use to find the determinant and the three product terms, as represented in Figure 9.

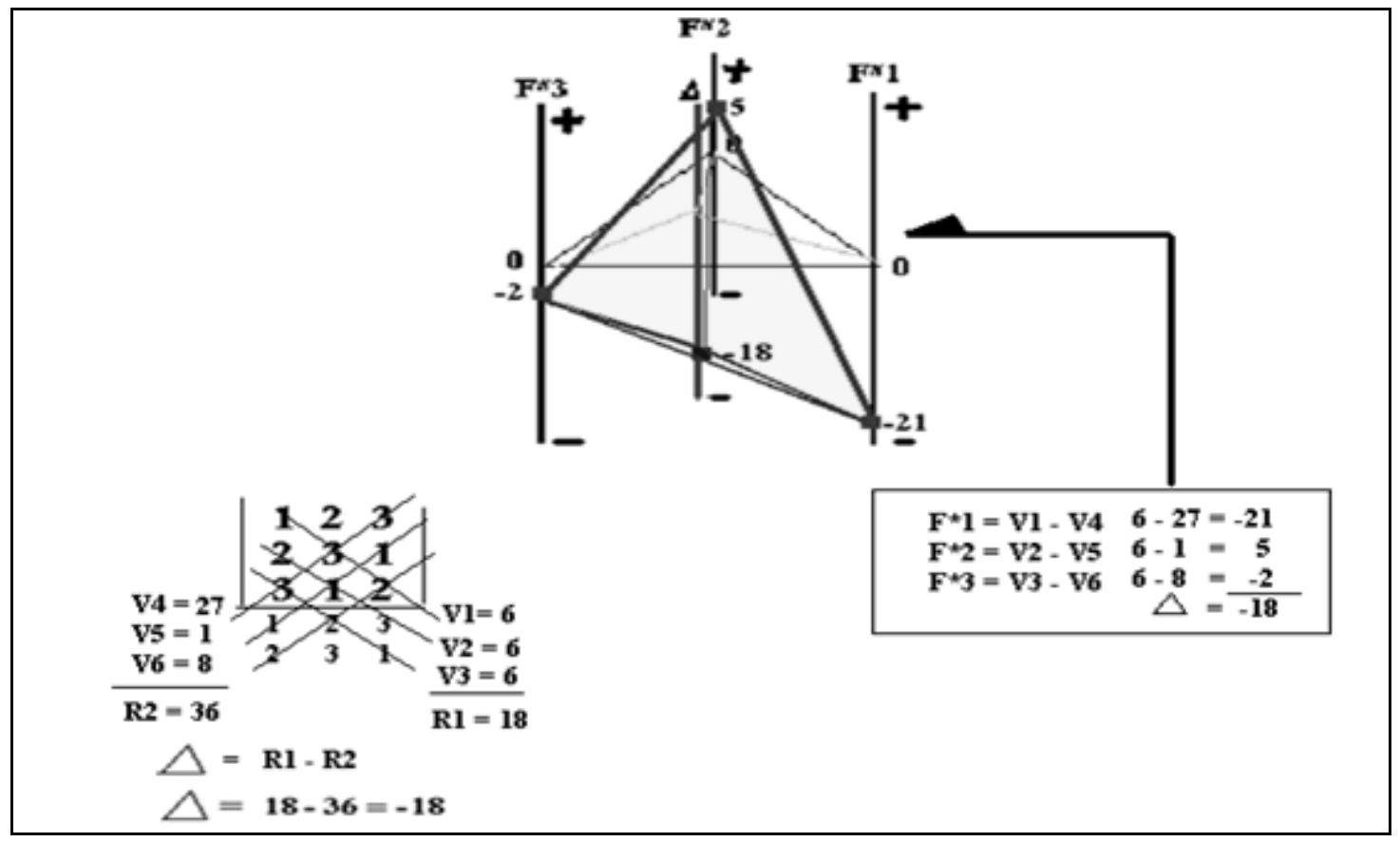

Figure 9: The Application of the Four Dimensional Coordinate System on the process to Plot The Determinant of a Matrix 3x3

\section{The Global Crisis Smash Graphic Effect Model (GCSGE-Model)}

\section{Introduction to the Global Crisis Smash Graphic Effect Model (GCSGE-Model)}

The construction of the global crisis smash graphic effect model (or GCSEG-Model) is based on the application of wave graphical modeling. To build each wave in our simulation, we suggest the simultaneous application of the interlink coordinate system and real time multidimensional graphical modeling. Initially, the GCSEG-Model uses n-number of variables " $E$ " in its analysis. Each variable has its general axis; at the same time, each general axis can have a large number of sub-axes. All these subaxes are interconnected by straight lines until they reach the last sub-axis. A reminder: each sub-axis runs with different multi-dimensional partial derivative(s) $(\partial \mathrm{Y} / \partial \mathrm{X})$ in real time ( The idea of applying a large number of partial differentiation(s) successively is to generate an effect of movement in our graphical modeling method.

According to the GCSEG-Model, each sub-axis is interconnected to its general axis by the application of the interlinking sub-axis system "Jt". The function of this sub-axes system is to join each sub-axis to the same general axis. Finally, all general axes and sub-axes are joined at all levels of analysis (see Figure 10) under the application of the fixed exponential " $\lambda$ " in different periods of time $(t+1)$. However, the assumption is that all sub-axes and the general axis are moving under the application of real time multidimensional graphical modeling " "र" (see Expression 1). We also suggest the application of the Omnia Mobilis assumption (Ruiz Estrada, 2011b) to help in the 'relaxation' of each sub-axis. The Omnia Mobilis assumption will reduce the use of the Ceteris Paribus assumption in this simulator. Finally, we can observe a large number of surfaces in permanent movement using the GCSEG-Model. The movement of these surfaces starts from the epicenter of the interlink coordinate system until the end of the last sub-axis within the same general axis. The real impact of this simulator is located on the last sub-axis (see Figure 10). The final analysis of the GCSEG-Model is based on the analysis of different surfaces displayed in different parts of the interlinked coordinate system (see Figure 11). 


$$
\begin{aligned}
& \lambda_{t+1}
\end{aligned}
$$

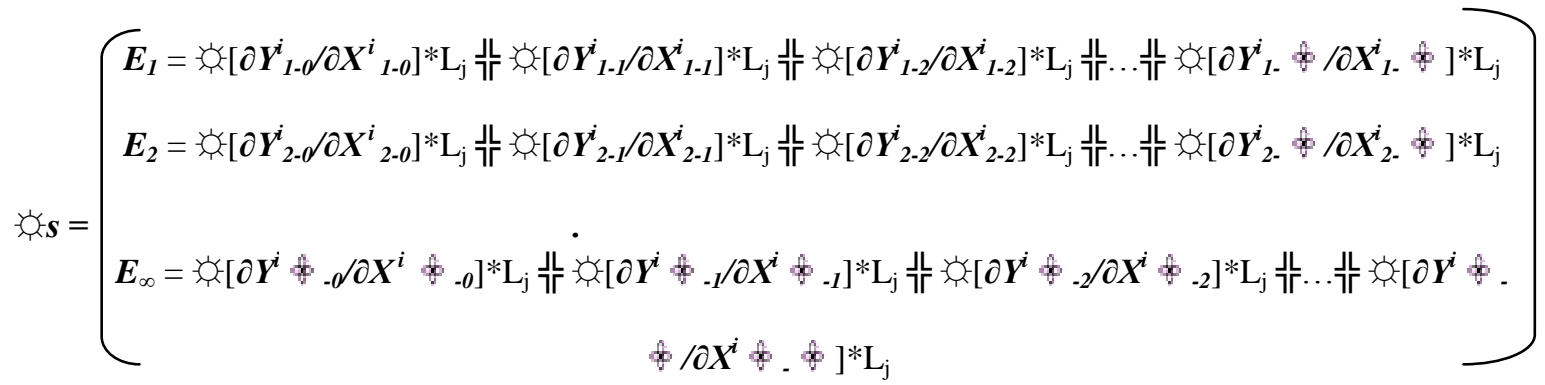

Partial differentiation: $\mathrm{i}=\{0,1,2,3 \ldots \notin\}$ and Level: $\mathrm{j}=\{\mathrm{o}, 1,2,3 \ldots \phi\}$

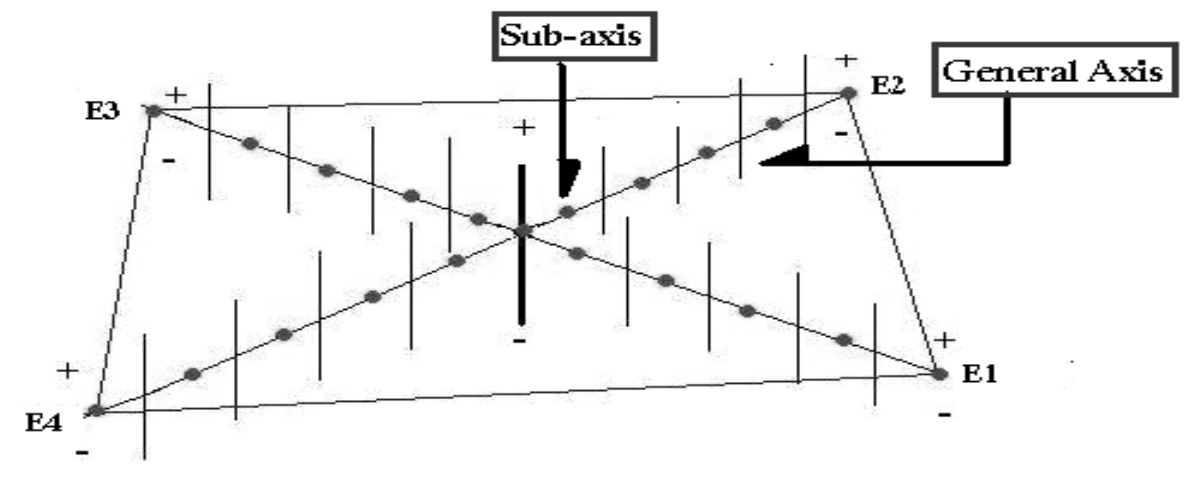

Figure 10: The Inter-linkage Coordinate System

Initial Level

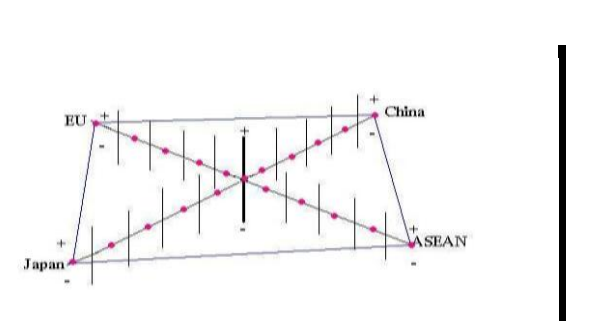

Level 7

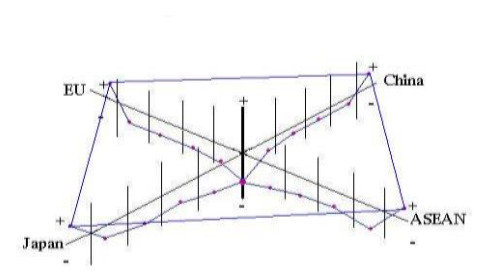

Level 10

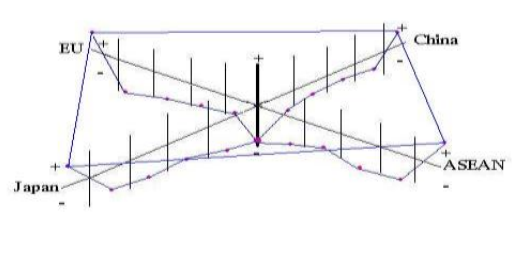

Level 5

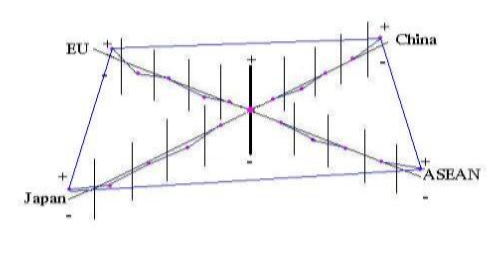

Figure 11: The GCSEG-Model: Graphical Modeling at Different Levels 
Level 6

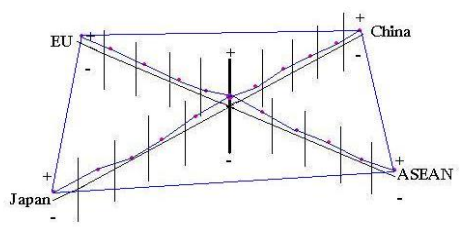

\section{Level 1}

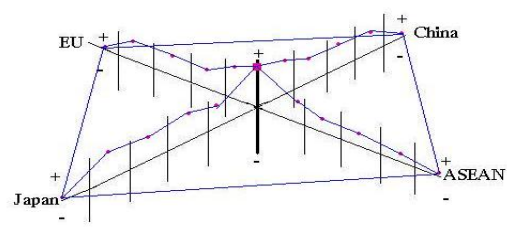

Figure 11 (cont): The GCSEG-Model: Graphical Modeling at Different Levels

\section{The Theory of Empty-Spaces}

\section{Introduction}

Initially, the theory of empty space is based on the use of the mega-space (see Appendix: Figure 2). According to this research, the mega-space is defined "as a multidimensional space formed by megasfinity spaces, where each space represents a specific dimension." Basically, we assume that each dimension is running at different speed of time. The concept of time in this research is totally different from the average concept of time, because here, time is a multi-dimensional variable and different types of time exist. Time is

\section{Level 3}

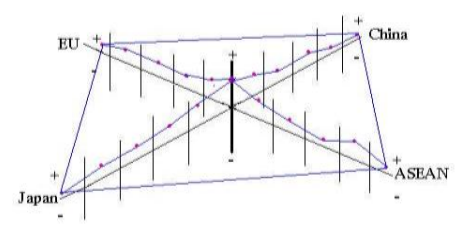

divided into three categories: (i) constant time (past time), (ii) partial time (present time) and (iii) chaos time (future time).

The quantification of time is made possible by the application of an infinite number of partial derivatives (see Expression 1) that are running in real time (s) within a specific space (see Expression 2). At the same time, this research introduces a new mathematical symbol called 'empty space,' which is represented by “圈”. The empty space symbol represents a specific space in the mega-space that interacts with different type of time.

$$
\begin{aligned}
& \partial \alpha_{0: 0: 0} \partial \partial \beta_{0: 0: 0} \ldots \partial \alpha_{\infty: \infty ; \infty} / \partial \beta_{\infty: \infty: \infty} \Rightarrow \lim \mathrm{i} \rightarrow \infty ; \lim \mathrm{j} \rightarrow \infty \& \lim \mathrm{k} \rightarrow \infty(1)
\end{aligned}
$$

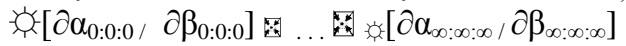

The coordinate system used in the theory of empty space is based on the application of Expression 3:

$$
\left(\left[\alpha_{\mathrm{i} j \mathrm{j}: \mathrm{k}}\right],\left[\beta_{\mathrm{i} \mathrm{j}: \mathrm{k}}\right]\right)
$$

Similarly,the measurement of any empty space in the mega-space is made possible by the application of Expression 4. We can observe that the integration of four partial derivatives exists over the use of four equations that run in real time all the time. Firstly, we assume that any empty space is located between $[-1,0)$ And $(0,1]$.
At the same time, we apply four vectors to its external coefficients $(\lambda)$. Additionally, each partial derivative in real time applies an extra exponential coefficient ( runs in real time simultaneously to neutralize the partial derivatives. This helps to neutralize the speed of the four partial derivatives at the same time. It can also help in the final measurement of each empty space " within the mega-space with higher accuracy. 


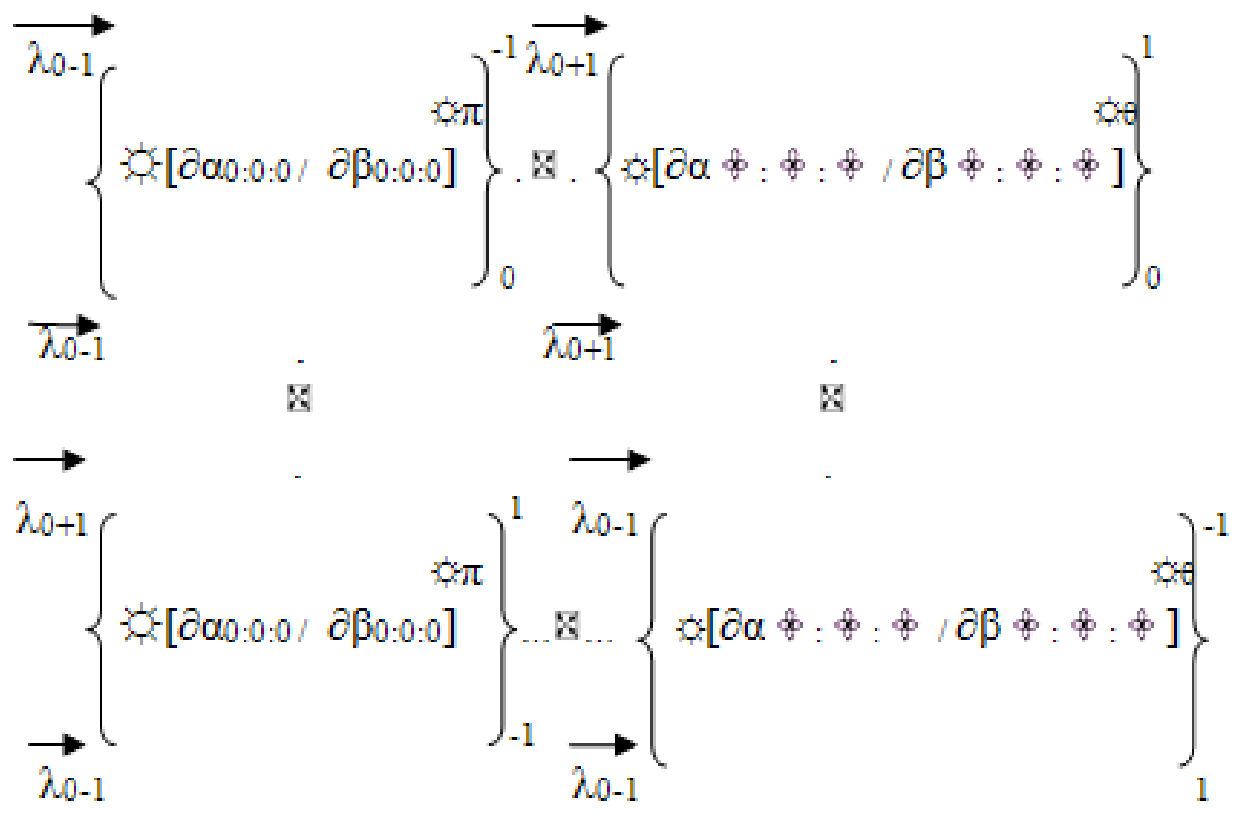

Therefore, we can simplify Expression 4 using Expression 5. This is made possible by simplifying the four equations by using only two

descriptive equations at the initial and final stage to measure any empty space.

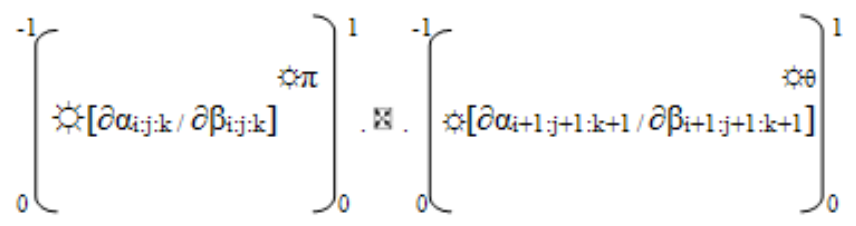

In fact, if we replace values from zero until infinity into Expression 5, it is then possible to observe, in Expression 6, the interaction of six inter-face equations that interact together at different levels of analysis at the same time.

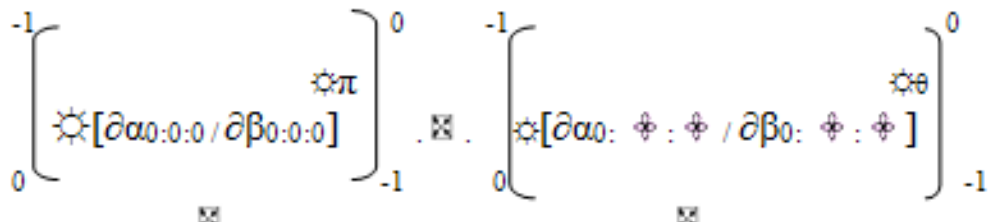

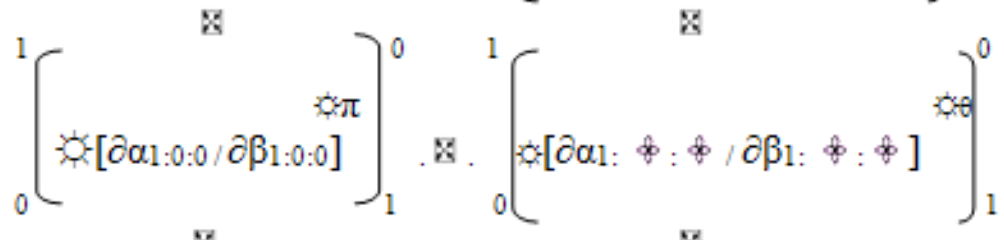

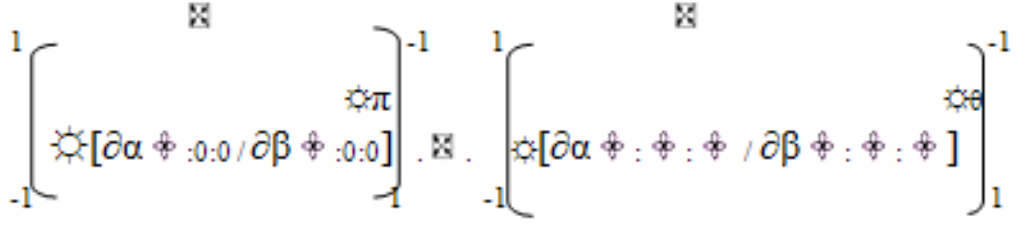


Finally, the quantification of the final empty space " $"$ within the mega-space is made possible by the application of Expression 7. The last expression clearly shows that the four partial derivatives $(\partial)$ that run in real time (os) under different levels can quantify any empty space in the mega-space without any restrictions. Expression 7 is derived from Expressions 1, 2, 3, 4, 5 and 6. Expression 7 applies arrows that are called "empty space marginalization," which consist of joining the four equations located in each corner of the empty space. At the same time, the measurement of empty space applies the inter-connectivity of multi-dimensional coordinate systems (卢) to join the four partial derivatives in real time to show final results.

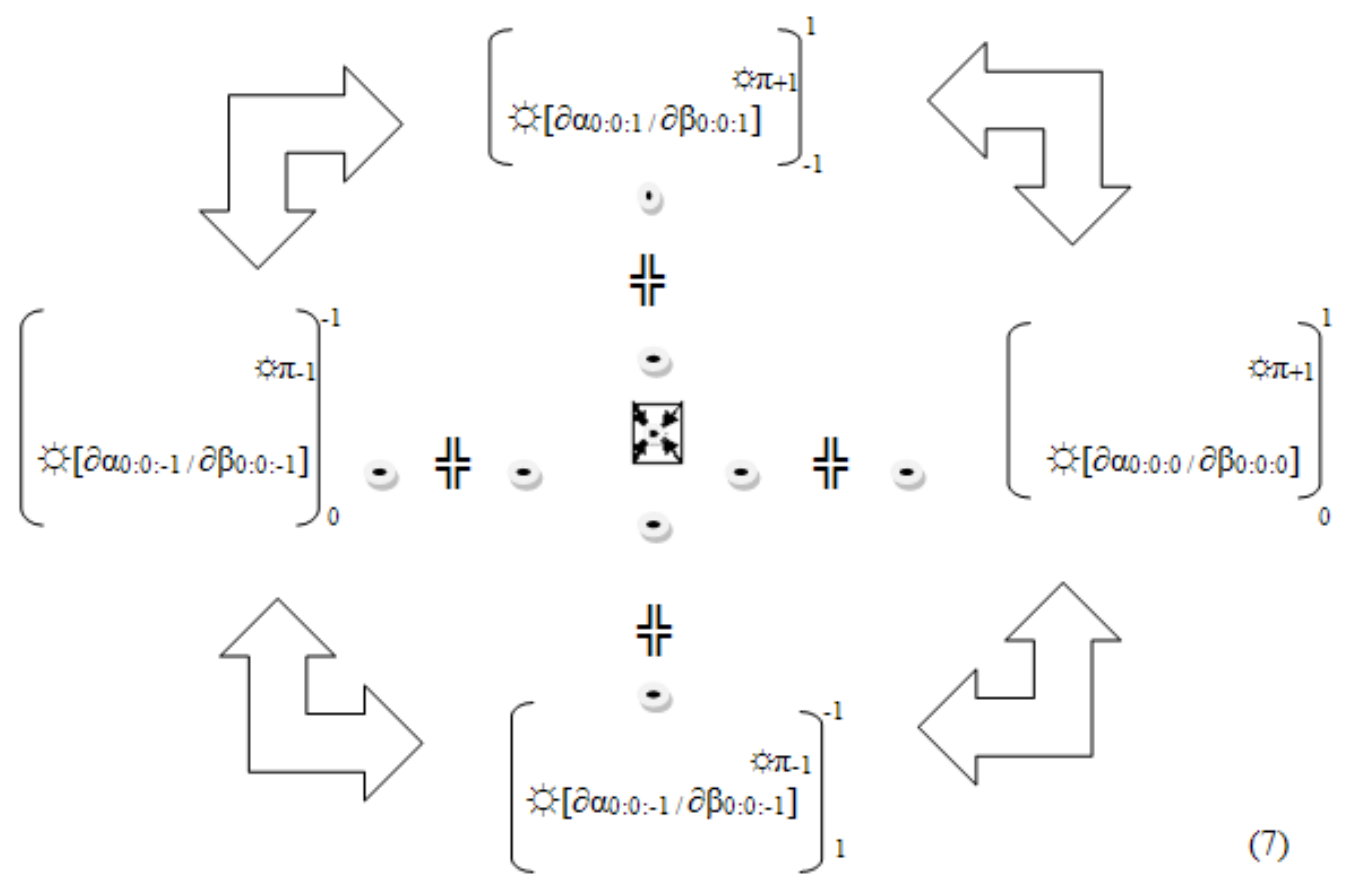

The Mega Time-space Continuum

Introduction: A Short Review of the Minkowski 4-dimensional Time-Space Concept

Initially, before we start reviewing the Minkowski 4-dimensional time-space concept or the "World" according to Hermann Minkowski (1907), it is necessary to review the classic idea about time and space as put forward by Euclidean geometry (Arfken, 1985, Dodge, 2004). Firstly, Euclidean geometry needs to be divided into two large sections: 2-dimensional Euclidean geometry and3-dimensional Euclidean geometry. 2dimensional Euclidean geometry uses the application of plane geometry (Klee, 1979) and 3-dimensional Euclidean geometry applies solid geometry (Altshiller-Court, 1979).

2dimensional Euclidean geometry is based on the use of a single plane that is located in two dimensions, but if this plane is displayed on a higher-dimensional level, it is possible to observe a hyper-plane in n-dimensions. At the same time, the calculation and intersection of different angles within the same plane make it possible to build a dihedral angle according to 2-dimensional Euclidean geometry. Finally, plane geometry offers us a series of different geometrical figures such as lines, circles and different type of polygons with different numbers of sides within the same graphical space simultaneously.

The study of the time-space continuum using the general theory of relativity from a geometrical perspective is by Albert Einstein; it demonstrates an alternative approach to understanding the structure of the Universe. Basically, Einstein applied two theoretical geometrical frameworks for the study of the time-space continuum. These two theoretical geometrical frameworks are Gaussian coordinates and Minkowski's 4dimensional time-space concept. Firstly, Gaussian coordinates are based on the use of a space of an arbitrary group of curves: u-curves that start from $\mathrm{u}=1, \mathrm{u}=2$ until $\mathrm{u}=\infty$, and $\mathrm{v}$-curves 
that start from $v=1, v=2$ until $v=\infty$. Both sets of curves are united within the same graphical space (see Figure 12) and finally; the union of both set of curves can build a single surface within the same graphical space. Hence, the Gaussian coordinate system is made up of the coordinate system $(\mathrm{u}, \mathrm{v})$.

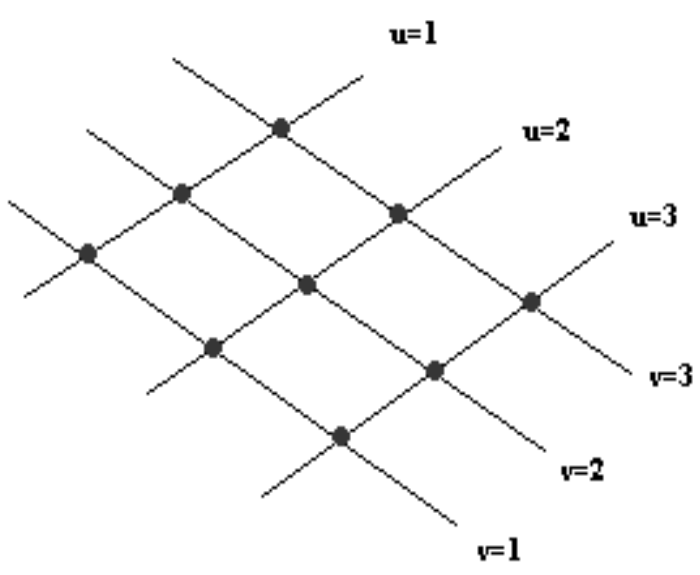

Figure 12: Gaussian Coordinates

The 3-dimensional Euclidean geometry is the opposite of 2-dimensional Euclidean geometry. 3-dimensional Euclidean geometry is focused on the construction of 3-dimensional geometrical figures through the use of spheres and polyhedral structures. In the case of the spheres, we use the interconnection of a set of points under different radii from its point of origin. But the use of polyhedral structures is based on the unification of polygons joined together by different edges into the same graphical space. We can observe that 2-dimensional Euclidean geometry and 3dimensional Euclidean geometry show different 2-dimensional and 3-dimensional geometrical figures by the use of 2-dimensional and 3dimensional coordinate systems respectively. Therefore, the idea of time and space across 2dimensional Euclidean geometry and the 3dimensional Euclidean geometry can facilitate an easy understanding of space with the construction of different geometrical figures that can give a general idea about time and space from different dimensions. The contribution of 2-dimensional
Euclidean geometry and 3-dimensional Euclidean geometry is great in the sense that it helps to understand and visualize different graphical spaces across different periods of time. However, we also need to acknowledge the contribution of the Minkowski 4-dimensional space-time concept to the understanding of time and space. Basically, this mathematical framework tries to explain the existence of four dimensions within the same space. Minkowski proposes four coordinates: $X_{1}$, $\mathrm{X}_{2}, \mathrm{X}_{3}$ and $\mathrm{X}_{4}$. Actually, Minkowski applies traditional 3-dimensional Euclidean geometry with the addition of one more coordinate that is $\mathrm{X}_{4} . \mathrm{X}_{4}$ represents the time value, where this time value is an imaginary number $\sqrt{ }-1$. In fact, we can figure that the relationship is closed for any event in the case of the $X_{1}, X_{2}$ and $X_{3}$, but for $X_{4}$ (time value), it is possible to observe some independency at certain levels compared to $X_{1}$, $X_{2}$ and $X_{3}$. However, in the case of the natural laws, we assume that the $\mathrm{X}_{4}$ coordinate keeps to the same level as $X_{1}, X_{2}$ and $X_{3}$ within the same graphical space supported by equations 1 and 2 (Einstein, 1952):

$\mathrm{X}_{1}{ }^{, 2}+\mathrm{X}_{2}{ }^{, 2}+\mathrm{X}_{3}{ }^{, 2}+\mathrm{X}_{4}{ }^{, 2}=\mathrm{X}_{1}{ }^{2}+\mathrm{X}_{2}{ }^{2}+\mathrm{X}_{3}{ }^{2}+\mathrm{X}_{4}{ }^{2}$

$$
\mathrm{X}_{1}{ }^{2}+\mathrm{X}_{2}{ }^{, 2}+\mathrm{X}_{3}{ }^{2}=\mathrm{X}_{1}{ }^{2}+\mathrm{X}_{2}{ }^{2}+\mathrm{X}_{3}{ }^{2}
$$

The Minkowski 4-dimensional space-time concept can be also called the 4-dimensional manifold. Actually, the 4-dimensional manifold is represented by a single surface that it is displayed in certain coordinate systems in a determinate period of time. A reminder: the Minkowski 4dimensional space-time concept never proposes a specific 4-dimensional coordinate system to model any manifold, it merely offers a diagram for the use of the classic 3-dimensional coordinate system and the addition of an extra coordinate that is called the world line (X4). The world line is not more than the time value that keeps constant under the use of an imaginary number $\sqrt{ }-1$. The world line is a straight line with a slope of $45^{\circ}$ that crosses into the origin of the 2 dimensional coordinate system (see Figure 13). 


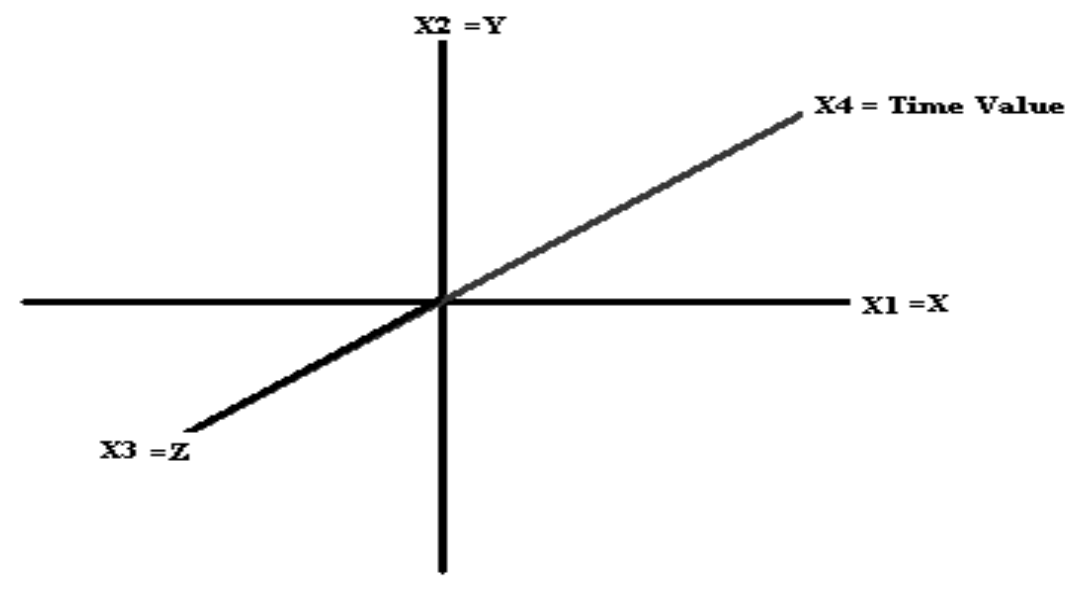

Figure 13: Minkowski Diagram

This is Albert Einstein's statement about Gaussian coordinates: "It is clear that Gauss coordinates are nothing more than an association of two sets of numbers with the points of the surface considered, of such a nature that numerical values differing very slightly from each other are associated with neighboring points 'in space"'. In fact, the idea of applying Gaussian coordinates into the general theory of relativity is that: "All Gaussian coordinate systems are essentially equivalent for the formulation of the general laws of nature" (Einstein, 1952). Therefore, the idea of time and space is the same phenomenon in the Universe. This proposition has changed all the traditional paradigms about time and space up to today.

However, Albert Einstein also applied a second geometrical approach based on Minkowski's idea of proposing the study of the 4-dimensional timespace continuum or World. Minkowski's 4dimensional space or World is basically a mathematic framework without any specific graphical coordinate system. Minkowski suggests the use of $X_{1}=x, X_{2}=y, X_{3}=Z$ and $X_{4}=V_{-} 1$, where $X_{4}$ is an imaginary number represented by $\sqrt{ }-1$. This is to give it the same form as the system of $\mathrm{X}_{1}, \mathrm{X}_{2}$, and $\mathrm{X}_{3}$ coordinates. In fact, Einstein always clarified that the time-space continuum of the general theory of relativity is not on Euclidean Continuum. Finally, after we analyze the theory of relativity from a geometrical perspective, we find that the infinity time-space coordinate system is able to give geometrical support to the general theory of relativity by introducing a multidimensional coordinate system to analyze the time-space continuum from a multidimensional perspective.

This short review about Minkowski's concept of 4-dimensional time-space provides the opportunity to comment briefly on this method of geometrical modeling, First, our conception about the time value or $\mathrm{X}_{4}$ is not an imaginary number $V_{-}-1$; it is instead a real number in $n$-dimensions with real coordinates $\mathrm{R}^{\mathrm{n}}$ (rational or irrational numbers). Therefore, we propose a set of multidimensional coordinate systems (see Figure 2) that are able to represent the 4-dimensional space $\mathrm{R}^{4}$ until infinity-dimensional space $\mathrm{R}^{\infty}$. In fact, the series of multidimensional coordinate systems proposed in this work accounts for all possible coordinates without any exception or the use of imaginary numbers $\sqrt{ }-1$ such as in the case of Minkowski's concept of 4-dimensional timespace.

Second, the idea of time and space in our case is totally different from the common perception of it, because time is not considered a single linear variable. Instead, it is an infinite series of multinon-linear variables that run at different levels of speed. This is because our basic premise is that space is multidimensional, where each space within the mega space maintains its own speed of time. In fact, we divide time into three different types: constant time "T-1" (past time), partial time " $\mathrm{T}$ " (present time) and chaos time " $\mathrm{T}+\mathrm{1}_{\mathrm{s}}{ }_{\mathrm{s}}$ " (future time). Constant time maintains a constant behavior in different spaces. Partial time is sporadic and imperceptible in different spaces. Finally, chaos time can appear at any time without any logical behavior within different 
spaces in the same graphical space. For example, we can imagine that the time we left behind in the past (constant time) has taken up some specific space and delineates some historical event(s) in a determinate period of time within the space. But if we move to the present time (partial time) that is happening at this specific moment in any space, we can observe that time always maintains a partial behavior. When we refer to the future time that we call chaos time, it is possible that it can appear at any time in different spaces simultaneously. Hence, for each dimension from the 4-dimensional space " $\mathrm{R}$ " until the infinitydimensional space " $\mathrm{R}$ " can experience different speeds of time such as constant time, partial time and chaos time.

Third, we can use multidimensional coordinate systems (Ruiz Estrada, 2011a) such as the Pyramid coordinate system, the Diamond coordinate system, the 4-dimensional coordinate system, the 5-dimensional coordinate system, the infinity-dimensional coordinate system and the Multi-functional Pictorial coordinate system (see Figure 2). These multidimensional coordinate systems help us to visualize different and unknown dimensions that are mentioned in Euclidian geometry and Minkowski's 4dimensional concept space-time has moved from being conceptualized through a formal mathematical theoretical framework to multidimensional graphical modeling. These six multidimensional coordinate systems are constructed based on the traditional 3-D space concept, but they can represent 4 dimensions $\left(\mathrm{R}^{4}\right)$, 5-dimensions $\left(\mathrm{R}^{5}\right)$, 8-dimensions $\left(\mathrm{R}^{8}\right)$, 9dimensions $\left(R^{9}\right)$ up to an infinity-dimensions $\left(\mathrm{R}^{\infty}\right)$.

We would also like to mention that if we include more than 3 dimensions in the same graphical space for the addition of more coordinate(s), then these additional coordinates become subcoordinates (sub-dimensions) in the same graphical space according to our research findings. Therefore, the moment we start to include more than 3 dimensions in our graphical modeling, we immediately start to use subcoordinates to represent additional subdimensions within the same graphical space.

\section{The Mega Time-Space Continuum Coordinate System}

The Mega Time-Space Continuum Coordinate System (Ruiz Estrada, 2011a) is based on five premises. First, it it based on the premise that the Universe (U) is multi-dimensional (see Expression 1.1), and that the Universe is moving at the different speeds of time. Second, the mega time-space continuum coordinate system is divided by a large number of general-spaces -GE(see Expression 1.2), sub-spaces -SS- (see Expression 1.3), micro-spaces -MS- (see Expression 1.4) and JI-Spaces (see Expression 1.5). In fact, all GS, SS and MS are moving in real time (-) and apply the multi-integrated principle (卢). This is to join all spaces in the mega time-space continuum coordinate system simultaneously. Finally, the mega time-space continuum coordinate system is based on the integration of all general-spaces (GS), sub-spaces (SS), micro-spaces (MS) and JI-spaces. All these spaces are running at different speed of time. The different systems are identified by "i" $=\{1,2 \ldots \infty$ \} for general-spaces, "j" $=\{1,2 \ldots \infty\}$ for subspaces (SS), "k" $=\{1,2 \ldots \infty\}$ for all microspaces and using the sub-coordinates " $\lambda$ " \{ $1,2 \ldots \infty\}$ and " $\mu$ " $\{1,2 \ldots \infty\}$ simultaneously for JI-spaces.

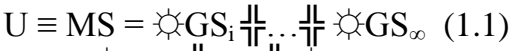

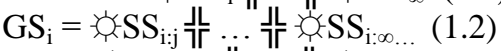

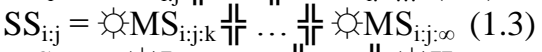

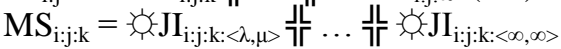

$$
\begin{aligned}
& \mathrm{JI}_{\mathrm{i}: \mathrm{j}: \mathrm{k}:<\lambda, \mu>}(1.5)
\end{aligned}
$$

Therefore, the mega time-space continuum coordinate system starts from general-space 0 (see Expression 1.6) to general-space infinity $\infty$ (see Expression 1.7):

$\mathrm{GS}_{0}=\left[\mathrm{SS}_{0: 0}, \mathrm{MS}_{0: 0: 0}, \mathrm{JI}_{0: 0: 0:<0,0>}\right] \quad(1.6)$

$\mathrm{GS}_{\infty}=\left[\mathrm{SS}_{\infty: \infty}, \mathrm{MS}_{\infty: \infty: \infty}, \mathrm{JI}_{\infty: \infty ; \infty: \infty<\infty, \infty\rangle}\right] \quad(1.7)$

The final general function to analyze the mega time-space continuum coordinate system is demonstrated by Expression 1.8:

$\mathrm{MS}=f\left(\mathrm{GS}_{\mathrm{i}}, \mathrm{SS}_{\mathrm{i}: \mathrm{j}}, \mathrm{MS}_{\mathrm{i} \mathrm{j}: \mathrm{k}}, \mathrm{JI}_{\mathrm{i}: \mathrm{j}: \mathrm{k}:\langle\lambda, \mu>}\right)$

The third premise is that the JI-space is a subcoordinate system $(\lambda, \mu)$ in the general coordinate system. This idea is based on the interception between $\lambda$-sub-coordinate and $\mu$-sub-coordinate within its micro-space respectively (see Expression 1.5).

The fourth premise is that the JI-space is a rigid body (or point) that is simply hanging or unfolding in its respective micro-space; in 
addition, two JI-spaces cannot occupy the same space at the same time. If we apply vectors among all JI-spaces together in the same microspace, then we can visualize a linear curve or non-linear curves moving in real time (to in micro-space.

The fifth premise is that the mega time-space continuum coordinate system (see Figure 1) is running at different speed and type of times. Initially, we have four types of time $(\pi)$ : constant time $($ past time $=\mathrm{t}-1)$, partial time $($ present time $=$ $\mathrm{t}$ ), chaos time (future time $\mathrm{t}+1$ ) and general time (Wt) (see Expression 1.9). General time is the synchronization of all general-spaces, sub-spaces, micro-spaces (see Figure 14) and JI-spaces within the same mega time-space continuum. Additionally, all systems in the mega time-space continuum are running at different speeds of time (see Expression 1.9); these depend on the location of each space and the distance from the point of origin.

\section{MEGA-SPACE}
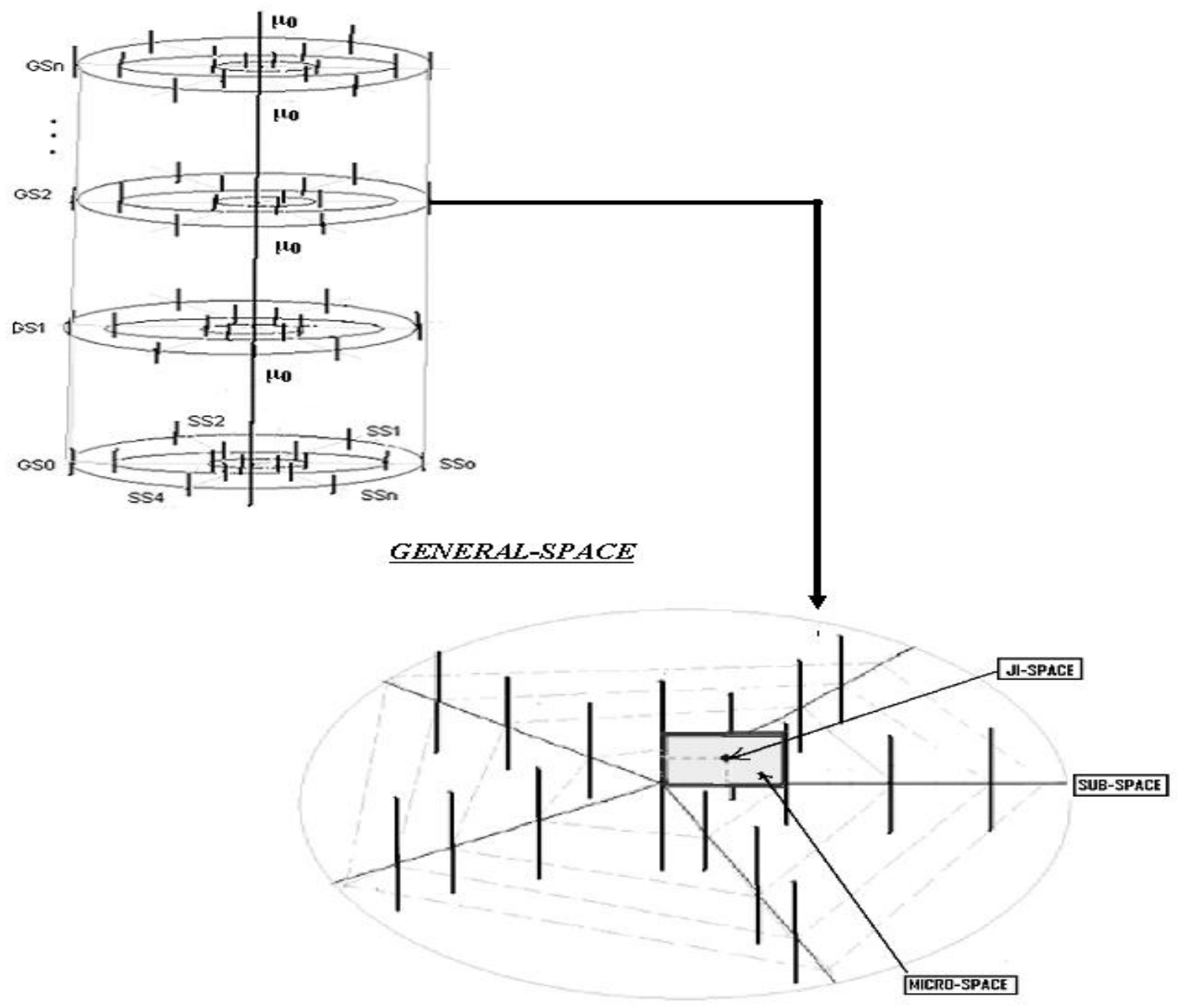

Figure 14: The Mega Time-Space Continuum

\section{An Introduction to the Concept of the Megasverses}

This section of this paper introduces the basic graphical and mathematical framework to explain the concept of the Megasverses. Initially, we assume that megasfinity ( $\phi$ ) exists. These are universes that are interacting, interconnected and working together in perfect harmony under different speed of time and levels of space. 
According to this research, megasfinity is equal to infinity raised to the power of infinity that is represented by $\infty^{\infty}$. We can consider the Megasverses to be a multidimensional group of mega-spaces that keeps in a constant state of evolution and transformation. Therefore, we can consider the Megasverses as a group of Megasfinity Universes, where each Universe is fixed in terms of its general-spaces (GS), subspaces (SS) and micro-spaces (MS) respectively. To support the idea of the Megasverses, we require the application of a special multidimensional coordinate system that can plot and map out the Megasverses. Hence, we suggest the application of the mega time-space continuum coordinate system.

The Megasverses can be mapped out using the mega time-space continuum coordinate system. It is possible to observe a large cylinder without end or specific size. It is possible to plot Megasfinity Universes into the mega time-space continuum coordinate system and it is possible to fix megasfinity general spaces (GS), sub-spaces (SS), micro-spaces (MS), nano-spaces (NS), JIspaces, JIM-spaces, $\alpha$-spaces, T-spaces and finally planets in each Universe. We would like to clarify that megasfinity sub-spaces (SS) are formed within each general space. At the same time, and in the same way, each sub-space has a megasfinity of micro-spaces (MS). Each microspace has a megasfinity of JI-spaces (JI). Similarly, a megasfinity of JIM-spaces exists in each JI-space, and each JIM-space has a megasfinity of $\alpha$-spaces, where in our case these $\alpha$-spaces can represent any Galaxy. Therefore, each $\alpha$-space has a megasfinity of $\mathrm{T}$-spaces that we can classify as a single solar system. Finally, we can find a large number of planets $(P)$ with different masses and sizes (see Figure 1) in each $\mathrm{T}$ space.

We assume that there always exists an unlimited number of "Big Bangs" in the Megasverses that keeps it in a state of constant expansion. While all universes keep in state of constant transformation and expansion without any borders or specific limitations, the different "Big Bangs" originate in different parts of the Megasverses that we would like to call the origin expansion points. Additionally, the idea of black holes in the Megasverses is represented by large tunnels that connect different universes in the same Megasverses. Everything absorbed by these black holes is transferred to another Universe. Objects are only transferred into another dimension or System, and perhaps changed into other forms; according to the principle of matter, matter can never truly be destroyed. Something important to mention at this point is that each Universe (GS) contains sub-spaces (SS), microspaces (MS), nano-spaces (NS), JI-spaces, JIMspaces, $\alpha$-spaces, T-spaces and planets. All these spaces are moving simultaneously under different speeds of time. Therefore, time can be divided into four different categories: constant time (pasttime), partial time (present-time), chaos time (future time) and general time. Hence, according to our research, the Megasverses is a multi-spacetime type of space. Finally, we can plot and locate any planet, solar system, galaxy or Universe using the mega time-space continuum coordinate system framework. The Megasverses coordinate system is expressed below:

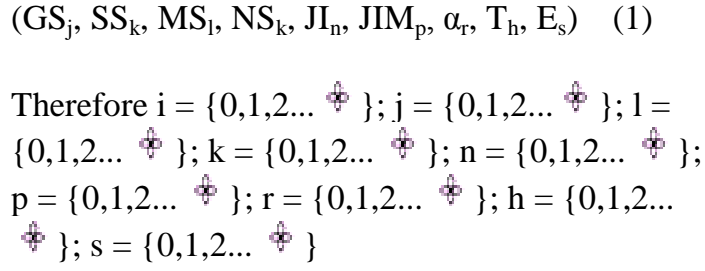

GS = Universe, $\mathrm{SS}=$ sub-space, $\mathrm{MS}=$ microspace, $\mathrm{NS}=$ nano-space, $\mathrm{JI}=\mathrm{ji}$-space, $\alpha$-space, $\mathrm{T}$ $=\mathrm{T}$-space and $\mathrm{E}=$ Planet

$$
\begin{aligned}
& \text { The general equation of the Megasverse is equal to: }
\end{aligned}
$$

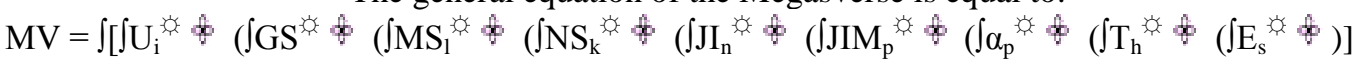

The general equation of Megasverses applies ten integrals that run in real time (-) simultaneously without end. At the same time, each space in the Megasverses carries different speeds of time, speeds of rotation and volume of energy. This means that a body located on the earth can experience ten different types of rotations simultaneously. Therefore, the earth does not only experience the single rotation around the sun. The earth also maintains different levels of rotation under higher levels such as generalspaces (GS), sub-spaces (SS), micro-spaces (MS), nano-spaces (NS), JI-spaces, JIM-spaces, $\alpha$-spaces, T-spaces that move in perfect harmony and synchronization. The rotation of any planet in its orbit is faster than the rotation of different galaxies in their general space (Universe). 


\section{An Introduction to Megasverses Gravitation}

Usually, when we refer to gravitation, the first thing to come to mind is the earth's force of attraction upon all objects. From a scientific point of view, gravitation was mainly studied by three scientists: Galileo Galilei (1623), Isaac Newton (1687) and Albert Einstein (1915). This research focuses on the study of the theory of general relativity (Rindler, 2001). Initially, the theory of general relativity is based on the use of the geometrical properties of time-space curvature (manifolds or surfaces). At the same time, the study of the time-space curvature is supported by the use of the Minskowski concept of 4dimensional space (Einstein, 1916) under the use of differential geometry and tensor calculus. In fact, the main idea of applying Minskowski's concept of 4-dimensional space was to explain that time and space is inseparable and complementary, and that this relationship can be represented by a wrap surface that is displayed on different levels in the Megasverses. Additionally, another concept crucial to support the general theory of relativity is the geodesic. The geodesic can be defined as the shortest curve between two points. Usually, the geodesic is explained in basic terms by a simple example: a body with a greater mass can attract bodies with less mass. According to Einstein, gravity is not a simple force if we examine the idea of the geodesic. It is a relationship between distinctive objects of different masses located in a specific time and space. Einstein's idea can be considered a crucial part of our research work. In fact, our proposition in this research is that Megasverses gravitation basically depends upon three conditions: the multidimensional geodesic position, the mass of the objects, and sustainable kinetic energy auto-generation. Therefore, the gravitation of any object in the Megasverses depends directly upon its multidimensional geodesic location at different levels in the Megasverses. At the same time, this research also incorporates the general theory of relativity, using mass effect and the time-space curvature. Additionally, this research supports the idea that any object in movement in the Megasverses can auto-generate its own long-term kinetic energy. Hence, this research proposes to combine the theory of general relativity, kinetic theory, and the higher multidimensional graphical modeling approach to explain the behavior of gravitation in the Megasverses. The theoretical framework of Megasverses gravitation is a simple extension of Einstein's general theory of relativity together with the application of kinetic theory (Landau and Lifschitz, 1976) and the use of a higher multidimensional graphical modeling approach. Initially, we would like to give an alternative definition of gravitation: "Gravitation can be defined as the perfect interaction of a megasfinity of multidimensional systems displayed at different levels in the Megasverses. At the same time, these multidimensional spaces permit any object to unfold and generate its own kinetic auto-energy for an unlimited time."

Hence, this section of this will give a short example about how gravitation works in the Megasverses. Our first premise is that any planet can experience several numbers of rotations at different levels in the Megasverses. Under Megasverses gravitation, any object can experience twelve different movements simultaneously at different speeds of time. This is the movement (rotations) of the Megasverses as a whole, general spaces (GS), sub-spaces (SS), micro-spaces (MS), nano-spaces (NS), JI-spaces, JIM-spaces, $\alpha$-spaces, T-spaces and planets (see Figure 2). Hence, geometrically, we can observe that any planet does not only experience the two usual forms of rotation: (i) upon its axis and (ii) around the solar system. We would like to point out here that the rotation of any planet in its multidimensional space depends upon its location in the Megasverses. From now on, we can account for the number of rotations $\left(r_{i}\right)$ that any object experiences in both short and long lapses of time. First, we can imagine that one object is located in any position on the earth and that: this object is moving in different directions $\left(\mathrm{r}_{1}\right)$; the earth is moving on its axis $\left(\mathrm{r}_{2}\right)$; and around the solar system $\left(r_{3}\right)$. Additionally, our solar system (T-space) is moving in its Galaxy ( $\alpha$-System) $\left(\mathrm{r}_{4}\right)$. Hence, in the same way, the Galaxy ( $\alpha$-System) is moving within its JIM-space $\left(\mathrm{r}_{5}\right)$; the JIM-space is moving in the JI-space $\left(r_{6}\right)$; the JI-space is moving in its nano-space $\left(r_{7}\right)$; the nano-space is moving in its micro-space $\left(\mathrm{r}_{8}\right)$; the micro-space is moving in its sub-space $\left(\mathrm{r}_{9}\right)$; each sub-space is moving in its general-space $\left(r_{10}\right)$; different general spaces are moving in the Megasverses $\left(r_{11}\right)$. Hence, any object located in the Megasverses can experience eleven rotations simultaneously under different speed of time. We can say that gravity is a universal stage that is the result of the simultaneous interaction of megasfinity multidimensional geodesics, different masses, and levels of kinetic autoenergies in the Megasverses. Megasfinity gravitation is represented by this equation: 


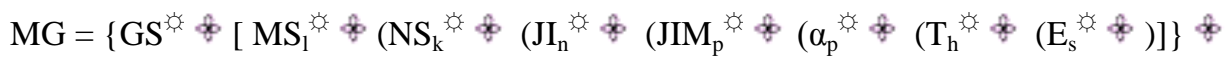

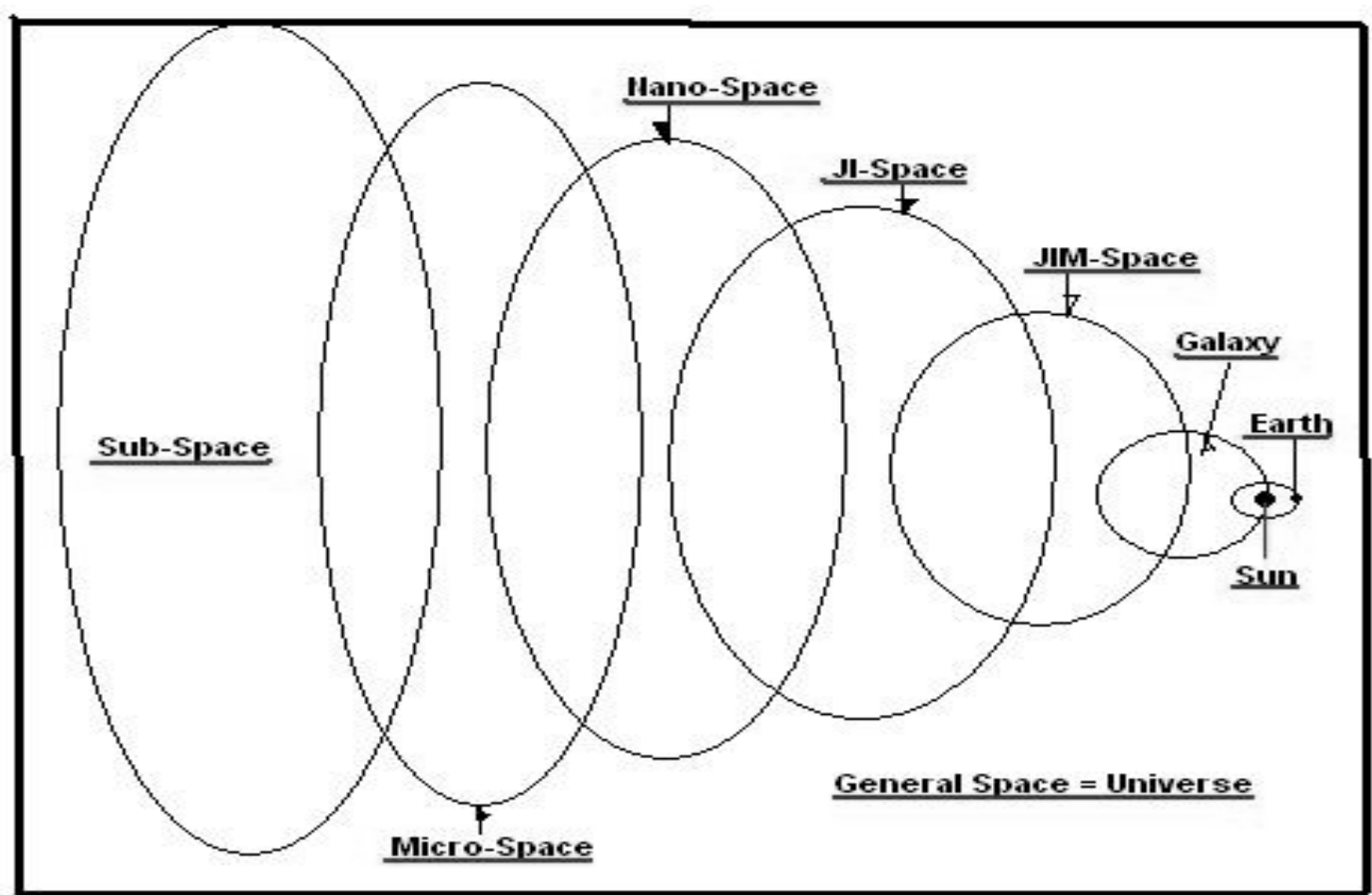

Figure 15: The Megasverses Rotation System

\section{CONCLUSION}

\section{MATHEMATICS}

We conclude that the case of the number zero " 0 " and infinity in multi-dimensional graphic modeling is totally different from 1dimensional, 2-dimensional and 3dimensional graphic modeling. We proposed that the number zero " 0 " is a large, single surface that is fixed in the mega-space. In the case of infinity, this research proposes that there exists one concept more complex than infinity, which is called megasfinity " $\phi$ ". Megasfinity " $\phi$ " is equal to the infinite exponential of infinity. This can be demonstrated by the application of multidimensional partial derivatives.

The Megasfinity ( $)$ supports the study of multidimensional coordinate systems. It allows us to understand unknown dimensions that were never explored by the 1-dimensional, 2-dimensional and 3dimensional coordinate systems. Finally, the use of the infinity coordinate system and the application of megasfinity demonstrate that there exist more than 3-dimensions.

This paper proposes an alternative mathematical framework for calculus. It is based on the introduction of the theoretical framework of multidimensional partial differentiation. Hence, we can analyze a large number of equations that interact together in different graphical spaces or dimensions within the infinity coordinate system.

Basically, this research concludes that it is possible to plot any matrix " $m$ " by " $n$ " in the same graphical space from basic operations such as addition, subtraction and multiplication of matrices until we can plot the determinant. This is only possible with the use of the mega-surface coordinate system. This method makes it possible to represent graphically a large system of simultaneous equations by matrices in the same graphical space and time. According to our findings, the visualization of matrices can help in the process of understanding the basic operations and behavior of matrices 
from a multi-dimensional graphical perspective.

Finally, this topic offers an alternative multi-dimensional graphical modeling approach to analyze the final impact of any global crisis from a multi-dimensional perspective. The GCGES-Model can generate a large number of scenarios originating from a global crisis, according to the level of devastation of the global crisis (L) coefficient. The main objective of this method of graphical modeling is to create different simulations and measure the catastrophic(s) effect(s) of any global crisis within the same graphical space.

\section{PHYSICS}

First, the study of dimension(s) is necessary to be studied through the empty space " within the mega-space. The main objective is to quantify different systems or dimensions that develop different speeds of time simultaneously in the same graphical space and time. In fact, the theory of empty space opens the possibility of offering a basic mathematical and graphical modeling approach to analyze unknown dimensions in the same graphical space and time, according to this research.

Second, the mega time-space continuum coordinate system can be used to plot timespace from a multidimensional perspective. The idea of time discussed in this research is totally different from the general conception of time because time is now divided into the different type of times that run in different parts of the mega timespace continuum. This means that each part of the mega time-space continuum coordinate system is running in its own speed of time. The use of multidimensional coordinates systems makes it possible to visualize unknown dimensions that the Minkowski 4-dimensional time-space concept proposes. Hence, according to our research, we find that the use of more than 3-dimensions or coordinates implies the use of sub-coordinates in the same graphical space. However, we also conclude that time is not a unique linear variable; instead, it is an infinite set of non-linear variables under different type of time, such as constant time, partial time and chaos time. This is because different speeds of time develop in different dimensions.

Finally, this paper concludes that is possible to map out different Universes using the mega time-space continuum coordinate system. With the application of the mega time-space continuum coordinate system, it is possible to locate different Universes distributed in different parts of the Megasverses. Additionally, this paper argues that the Megasverses maintains a state of constant expansion based on the emergence of a large number of simultaneous "Big Bangs" in different Universes. These black holes can be represented by a large number of tunnels that connect different Universes successively in the Megasverses. Finally, this research gives a new definition of gravitation in the case of Megasverses: "Gravitation can be defined as the perfect interaction of a megasfinity of multidimensional systems displayed at different levels in the Megasverses. At the same time, these multidimensional systems permit any object to unfold and generate its own kinetic energy for an unlimited time."

\section{REFERENCES}

1. Altshiller-Court, N. (1979). Modern Pure Solid Geometry. New York: Chelsea.

2. Arfken, G. (1985). Mathematical Methods for Physicists, 3rd ed. Orlando, FL: Academic Press, pp. 198-200.

3. Dodge, C. W. (2004). Euclidean Geometry and Transformations. New York: Dover.

4. Einstein, A. (1952). Relativity: The Special and the General Theory. New York: Three Rivers Press.

5. Goldstein, H. (1980). "The Euler Angles" and "Euler Angles in Alternate Conventions." §4-4 and Appendix B in Classical Mechanics, 2nd ed. Reading, MA: Addison-Wesley, pp. 143-148 and 606-610. 
6. Klee, V. (1979). "Some Unsolved Problems in Plane Geometry." Math. Mag. 52, pp. 131-145.

7. Landau, L. D. and Lifschitz, E. M. (1976) Mechanics, 3rd ed. Oxford, England: Pergamon Press.

8. Rindler, Wolfgang (2001). Relativity: Special, General and Cosmological. Oxford University Press.

9. Ruiz Estrada, M. A. (2011a) "Multidimensional Coordinate Spaces" International Journal of Physical Sciences, 6(3):340-357.

10. Ruiz Estrada, M. A. (2011b). "Policy Modeling: Definition, Classification and Evaluation" Journal of Policy Modeling, 33(4):523-536.

11. Shchepin, E.V. (1998). "Topology of limit spaces with uncountable inverse spectra”, Uspehi Mat. Nauk 31, 5(191): 191 - 226.

12. Tengmark, M. (2003). "Science and Ultimate Reality: From Quantum to Cosmos". Cambridge University Press. 